\title{
Abhängigkeit der metamorphotischen Kiemenrückbildung vom Gesamtorganismus der Salamandra maculosa.
}

\author{
Von
}

\section{Werner Kornfeld.}

(Aus der Biologischen Versuchsanstalt der kais. Akademie der Wissenschaften in Wien, Zoologische Abteilung 1).)

Mit 3 Figuren im Text and Taf. IX und $\mathrm{X}$.

Eingegangen am 5. April 1914.

In einer vorläufigen Mitteilung (1913) berichtete ich über die Resultate von Versuchen über Kiementransplantationen an Salamanderlarven. Ich hoffte damals, daß es mir bald möglich sein wirde, einige ergänzende Versuchsreihen und histologische Untersuchungen durchzuftibren und dann eine genaue Darstellung des gesamten Erscheinungskomplexes zu bieten. Leider machen mir aber andere Arbeiten eine Vervollständigung der Versuche in dem gedachten Sinne vorderhand unmöglich. Um nun die Mitteilung der näheren Beweise für die damals aufgestellten Behanptungen nicht unnötig lange zu verzögern, sehe ich mich genötigt, meine Befunde schon jetzt rorzubringen, obwohl ich mir des Umstandes wohl bewußt bin, daß zu einer kausalen Durcharbeitung der aufgegriffenen Fragen noch eine Reihe wichtiger Untersuchungen fehlt.

Die Versuche wurden in der zoologischen Abteilung der Biologischen Versuchsanstalt in Wien in der Zeit vom September 1912

1) Diese Arbeit wurde nnter dem Titel: Mitteilnngen aus der Biologischen Versuchsanstult der kaiserl. Akademie der Wissenschaften, Zoologische Abteilung, Vorstand H. PrzmbaMr. 3. Abhïngigkeit der metamorphotischen Kiemenrückbildung vom Gesamtorganismus der Salamandra maculosa von Werner KoRNEELD, im Akademischen Anzeiger Nr. VIII angezoigt (eine vorlüufige Mitteilung wurde bereits im Biolog. Zentralblatt. Bd. 33. 1913 veröffentlicht). 
bis Dezember 1913 durchgefthrt. Es sei mir gestattet, an dieser Stelle dem Leiter der Abteilung; Herrn Prof. Dr. Hans Przibram, fur die Überlassung eines Arbeitsplatzes sowie für mannigfache Ratschlige und Anregungen meinen herzlichsten Dank auszusprechen. Zu besonderem Danke bin ich ferner. Herrn Dr. Eduard Uhuenhuth verpflichtet, dessen Arbeiten ỉber Transplantation des Amphibienauges den Anstoß zu den vorliegenden Untersuchungen gaben und der meine Arbeit stets durch auBerordentlich wertvolle praktische und theoretische Winke förderte.

\section{Die Literatur über experimentell nachgewiesene Abhängigkeit der Organe vom Gesamtorganismus.}

Die mannigfachen wechselseitigen Beziehungen des Gesamtorganismus und seiner Teile bieten der experimentellen Biologie eine ebenso mannigfache Reihe von Problemen, deren Lösung mit besonderem Erfolg mit Hilfe der Transplantationsmethode versucht wird. Es ist gelungen, auf diesem Wege eine Reihe von Beeinflussungen, die man schon nach dem normalen Verhalten der Organismen vermuten mußte, mit Sicherheit nachzuweisen und andere bis dahin noch unvermutete neu aufzudecken. In anderen Fällen wieder haben es negative Ergebnisse des Experimentes wabrscheinlich gemacht, daß sich die Organe in den betreffenden Punkten auch unter den natirlichen Bedingungen selbständig, vom Gesamtorganismus - d. h. von der Gesamtheit der ibrigen Organe - unbeeinfluBbar verbalten.

Solche Fälle scheinen vor allem bei allen systematisch charakterisierenden Eigenschaften vorzuliegen. Ich erinnere hier an die Verhältnisse bei der gärtnerisch verwendeten Pfropfung; die in den meisten Fällen (scheinbare Ausnahmen sollen später noch besprochen werden) zeigen, daß das Pfropfreis durch den Grundstock in seiner systematischen Natur nicht beeinfluBt wird (s. besonders Vöcrrisg 1892). Dasselbe zeigen auch zahlreiche Versuche auf zoologischem Gebiete. Vereinigungen von Stücken verschiedener Regenwurmarten weisen keine Änderung des Art- oder Rassencharakters in den Teilsticken auf, ebensowenig Vereinigung von Teilen verschiedener Varietäten von Haarsternen, verschiedener Arten von Fröschen $u$. a. Auch bei der Regeneration verhalten sich die vereinigten Teilsticke vollkommen selbständig voneinander. (Eine Zusammenstellung derartiger Versuche und ausfuhrliche Literaturangaben finden sich bei Korscheut 1907 und bei Schöne 1912.) 
Auch innerhalb derselben Art und Rasse behalten Gewebe, die von einer Region des Körpers in eine andere (auf dasselbe Individuum oder auf ein anderes) verpflanzt werden, die fur ihre ursprtingliche Lage charakteristische Eigenart bei. So verändert nach MarCHAND (1901, zit. nach SchöNe 1912) an Stelle von Nasenhaut verpflanzte Armbaut ihren Charakter nicht. Die Eigenart von Haaren der Bauchhaut von Mänsen bleibt, auch wenn sie auf den Rücken verpflanzt wird, unverändert (ScHöN 1912). Bei diesen Versuchen zeigte sich auch der Haarstrich unabhängig davon, in welcher Richtung im Verhältnis zur Längsachse des Empfängers das transplantierte Stiick aufgelegt wurde. Umgedrehte Hautstiicke behielten ibren nun dem der Umgebung entgegengesetzten Haarstrich unverändert bei. Auch lokal begrenzte Bildungspotenzen jugendlicher Gewebe werden im allgemeinen durch Transplantation nicht abgeändert.

Abweichend lauten einige Angaben über die Pigmentierung transplantierter Hant. Während diese nämlich in vielen Fällen ebenfalls vom Wirtstier unabhängig bleibt (so WINIKLer 1910, WEIGL 1913), werden in andern Fällen Änderungen der Färbung des Transplantates unter dem Einfluß der umgebenden Haut des Wirtstieres angegeben. (Solche Befunde sind beispielsweise zusammengestellt bei Weigl 1913). Es ist aber ausdricklich zu betonen, daß, auch wenn solche Beeinflussung in bestimmten Fällen mit Sicherheit nachgewiesen sein sollte, diese Tatsache mit den früher erwähnten in keinerlei Widerspruch sttunde. Denn es könnte auch hier nicht eine Änderung der wesentlichen eigenen Charaktere der übertragenen Gewebe, sondern nur ein Ein- oder Auswandern von Elementen vorliegen. Daß derartiges rorkommt, zeigen zahlreiche Befunde. Das prägnanteste Beispiel sehen wir wohl bei den als Chimären erklärten Pfropfbastarden; dort erfolgt eine scheinbare Beeinflussung des Pfropfreises durch den Grundstock dadurch, daß von diesem aus Gewebe das Pfropfreis durchwuchern oder umwachsen. Es kann so das Pfropfreis in bezug anf Färbung, Gestalt usw. scheinbar Charaktere annehmen, die eine Mittelstellung zwischen den Eigenschaften ron Grundstock und Pfropfreis einnehmen.

Ebenso verhält es sieh bei dem von Harrison (1904) beschriebenen Übergreifen der dunkeln Seitenlinie von einem Vorderleib einer Rana silvatica auf den mit ihm vereinigten hellen Hinterleib einer Rana palustris.

Auch Befunde von Crampton (1897, 1898 zit. nach Crampton 1899) über ein bei Vereinigung von Schmetterlingspuppen verschie- 
dener Art in Ansnahmsfällen stattfindendes Annehmen der Färbung der größeren Komponente durch die kleinere, dürfte in einem derartigen Durchtritt von Elementen an der Vereinigungsstelle seine Erklärung finden. Endlich scheinen auch bei den später noch zu besprechenden funktionellen Beeinflussungen des Transplantates in manehen Fällen Durchwachsungen eine Rolle zu spielen.

Außer geformten Elementen wandern auch Lösungen aus der einen Komponente in die andere ein. Die Lösungen der Nährsalze werden aus dem Grundstock in das Pfropfreis iibertragen, die Assimilate ans dem Pfropfreis treten in den Grundstock ein. Ebenso findet bei tierischen Objekten Übertritt der das Transplantat ernährenden Stoffe aus dem Wirtsorganismus statt. Ein Übertritt von nicht "plastischen * Stoffen ist im Pflanzenreich wiederholt angegeben worden. So beschrieb Guignard (1907) eine Wanderung der Blausäure liefernden Glykoside aus blansäurehaltigen in blausäurefreie Phaseolus-Arten. Die Beweiskraft seiner Untersuchungen wird allerdings jetzt angezweifelt. LindemuTer (1877) gab in einem Fall eine Wanderung eines Farbstoffes bei Vereinigung verschieden gefärbter Kartoffelrassen an. Doch erscheint es in diesem Falle zweifelhaft, $a b$ es sich nicht doch um eine in der scheinbar beeinflußten Komponente selbst stattfindende Farbproduktion handelte, die auch ohne Beeinflussung durch die andere Komponente event. auftreten könnte. Auch Vöchтім beschreibt einen ähnlichen Fall, den er aber selbst nicht als aus Beeinflussung durch die andere Komponente, sondern durch Farbproduktion anf den Wundreiz hin erklärbar findet. Endlich finden sich Angaben über Wanderung von Alkaloiden bei Moess (1882), Leersum (1900), Strasburger (1885 u. 1906), Grafe und Linsbauer (1906) und in einer ausfuhrlichen und wie es scheint sehr kritischen Arbeit von Meyer und Schurdo (1910).

Ein solches Übertreten von Stoffen können wir nattirlich auch noch nicht als Beeinflussung der einen Komponente durch die andere bezeichnen. Doch kann von den eingewanderten Stoffen nun ein Einfluß ausgehen, der eine Änderung in dem Gewebe der betreffenden Komponente selbst hervorruft. Von GRAFE und LiNsBaUer wurde beispielsweise angenommen, $d a B$ es sich bei ihren Resultaten nicht um ein einfaches Übertreten des Alkaloides handle, sondern daB in der einen Komponente eine ihr ursprtinglich fremde Alkaloidbildungsfähigkeit unter dem Einfluß der andern Komponente hervorgerufen wurde. Strasburger wieder schrieb dem tubertretenden Atropin eine Beeinflussung der Knollenbildung zu. Doch wurden 
die Resultate dieser beiden Arbeiten nicht allgemein als beweisend angesehen.

Der einfachste Fall einer wirklichen sicher festgestellten Beeinflussung ist die Beeinflussung der einen Komponente durch die von der andern her erfolgende Ernährung. Durch sie scheint vor allem die Dauer der Erhaltung eines Transplantates und sein Wachstum, in einigen Fällen aber auch sein Habitus, bestimmt zu werden. Als Beispiel hierftur möge wieder die Erfahrung der Gärtner dienen, daß ein Pfropfreis je nach der Unterlage einen verschiedenartigen Wuchs anfweisen kann, so etwa in einem Fall mehr strauchig, im andern Fall mehr baumartig erscheint. Diese Verschiedenheit durfte wohl auf die verschiedene Menge zugefulhrter Nährstoffe, sowie auf die quantitative Verschiedenheit der ftir den Stofftransport zur Verfugung: stehenden Kräfte zurlickzufuhren sein. Die Ernährung spielt jedenfalls auch bei der von Born (1897) gefundenen Tatsache eine Rolle, daß von zwei miteinander vereinigten Teilstücken von Froschlarven oft das eine an Größe zurïckbleibt. Die zugleich festgestellte, später noch zu besprechende Erscheinung, daß sich die beiden Teilstucke trotzdem genau gleichzeitig verwandelten, daß auch die einzelnen Entwicklungsschritte (beispielsweise das Hervorbrechen der Hinterbeine) gleichzeitig an beiden Stücken vor sich gehen, beweist, daß von dieser Beeinflussung der quantitativen Ausbildung der Teilsticke durch gtinstige oder ungünstige Ernährung das Entwicklungstempo wenigstens in diesem Falle nnabhängig war (daß also für die später zu besprechende "Synchronie" der Entwicklung andere Faktoren verantwortlich sein müssen).

Ernährung durch das Wirtstier könnte auch bei der von KopEó (1911) festgestellten Tatsache, daß bei Schmetterlingen in Weibchen verpflanzte Hoden hypertrophieren, in Männchen verpflanzte Ovarien in ibrer quantitativen Ausbildung zurïckbleiben, eine Rolle spielen. (KopeÓ selbst erklärt die Erscheinung durch den im Männchen und im Weibchen verschieden großen Raum, der flir die Entwicklung der Gonade zur Verfügung steht. Auch in andern Fällen könnten solche mechanische Faktoren eine Wirksamkeit haben.)

Es erscheint außerordentlich interessant, daß auch Eigentlimlichkeiten des Wachstums, die, soweit sie innerhalb der systematischen Einheit labil sind, auch am Transplantat leicht durch Nahrungseinflüsse verändert werden können, soweit sie fur eine systematische Einheit charakteristisch sind, auch bei der Transplantation beibehalten werden. So wächst nach WeIGL (1913) Axolotlhaut auf Sala- 
manderlarven entsprechend ihrer Arteigentiimlichkeit schneller als die wirtseigene Salamanderlarvenhant, was sich erstens durch ein Überwuchern derselben zeigt und zweitens anch durch Messungen festgestellt wurde. Da nun die Ernährungsbedingungen, soweit es auf den Wirtsorganismus ankam, für das Transplantat doch sicher eher unguinstiger waren als fur die wirtseigene Haut, müssen wir hierin tatsächlich ein Festhalten einer systematischen Eigentumlichkeit erblicken, die uns von vornherein leicht beeinflußbar erscheinen konnte.

An die Einflusse der Nahrungszufuhr wären die anderer spezifischer Stoffe anzureihen, und zwar sei hier vor allem an die Wirksamkeit der inneren Sekretion erinnert. Diese spielt wohl eine größere Rolle bei der Frage nach der Beeinflussung des Gesamtorganismus durch seine Teile, bzw. des Wirtes durch das Transplantat. Doch wurde wenigstens eine Gruppe solcher Einflüsse auch als vom Gesamtorganismus dem Teil, vom Wirtsorganismus dem Transplantat weitergegeben, nachgewiesen. Bei der Transplantation von Organen, die sekundäre Geschlechtscharaktere aufweisen, konnte festgestellt werden, daß diese durch den Geschlechtscharakter des Empfängers beeinflußt werden. Durch die Gonadentransplantationen (s. beispielweise besonders STreinace 1912 u. 1913) ist es anderseits höchst wahrscheinlich gemacht worden, daß derartige Beeinflussungen ihre Ursache in einer inneren Sekretion vom interstitiellen Gewebe der Gonade her hat. Es können auf diesem Wege sowohl ständige Geschlechtscharaktere durch das Geschlecht des Empfängers beeinfluBt werden - so entwickelt sich nach BResKa (1910) der Riickenhautstreifen des Weibchens von Triton cristatus, auf das Männchen utberplanzt, zu dem fur dieses charakteristischen Kamme - als auch periodisch auftretende Charaktere durch den momentanen geschlechtlichen Entwicklungszustand des Empfängers. So ist nach L. LowB (1911) bei Uterustransplantationen am Meerschweinchen die Deciduabildung auBer rom Geschlechtszustand des spendenden Weibchens auch rom Geschlecht und vom Geschlechtszustand des Empfängers abhängig. Nach HARMs (1912) zeigen zurückgebildete Daumenschwielen kastrierter männlicher Frösche, auf normale briinstige Männchen verpflanzt, vollständige Aufdifferenzierung, während sich normale Daumenschwielen, auf Kastraten verpflanzt, zurlickbilden. Andere Fälle ftihren zu den später zu erörternden zeitlichen Beeinflussungen von Entwicklungsvorgängen hiniiber. So die von RrBbERT (1897) beschriebene Tatsache, daß bei der Transplantation der noch unausgebildeten Mamma eines Meerschweinchens auf das $\mathrm{Ohr}$, die 
Mamma sich dort zu einem zwar nicht ganz normalen aber doch funktionierenden Organ entwickelte, das genau zur selben Zeit wie die wirtseigenen Mammardrtisen Milch produzierte, nämlich als der Empfänger geworfen hatte. Die drei letzten Versuche zeigten auch, daß die Beeinflussung dureh die innere Sekretion nicht an den Ort gebunden sei, an dem die sekundären Geschlechtscharaktere normalerweise zur Entwicklung gelangen: HARMs verpflanzte die Daumenschwielen der Frösche auf die Dorsalseite des Kopfes, L. Lows die Uterusstucke in subcutanes Gewebe, Ribbert die Mammardriusen in Gewebe des Ohres, und trotzdem traten die besprochenen Beeinflussungen ein.

Zwei Gruppen von Erscheinungen sollen hier ganz kurz erwähnt werden, weil sie meist ebenfalls (so anch bei KorschelT 1907 und bei ScHöNe 1912) als Beeinflussungen des Transplantates durch den Wirtsorganismus behandelt werden. Die eine betrifft die angebliche Umkehrung der Polarität eines 'Transplantates unter dem Einfluß der entgegengesetzten Polarität des Wirtsgewebes. In Wirklichkeit scheint es sich bei den betreffenden Erscheinungen aber nicht um eine spezifische Beeinflussung des Transplantates durch den Wirt, sondern im Gegenteil um das gesetzmäBige Beibehalten einer sich auch sonst zeigenden, einseitig beschränkten Regenerationsfähigkeit zu handeln. Hierfür spricht vor allem die Tatsache, daß solche »Polaritätsumkehrungen * auch bei einfacher - nicht mit Transplantation verbundener - Regeneration vorkommen, sowie bestimmte GesetzmäBigkeiten bei diesen Erscheinungen, die ebenfalls ihre Identität mit normalen Regenerationserscheinungen wahrscheinlich machen. Immerhin wäre es möglich, daß einzelne Fälle sich dieser Erklärnngsweise nicht einfiugen. (Näheres über diese komplizierten Fragen siehe besonders bei PrzinRam 1909 u. 1913.)

Die zweite Gruppe von Erscheinungen ist die funktionsgemäße Umgestaltung des Transplantates, speziell bei Überpflanzung von Knochen und von Blutgefüßen. Venenstïcke erfahren, wenn sie an die Stelle von Arterienstücken verpflangt werden, eine Verdickung, die ihnen bald ein arterienähnliches Aussehen verleiht. Knochen erlangen, an Stelle anderer verpflanzt, die Struktur, die für die betreffende Stelle charakteristisch ist. Dabei aber scheint es sich in einigen Fällen nicht um eine Umwandlung der übertragenen Gewebe selbst, sondern um eine Verdrängung derselben durch mit funktionsgemäßer Struktur regenerierende Gewebe des Wirtsorganismus zu bandeln. In andern Fallen scheint allerdings eine solche Verdrän- 
gung durch Wirtsgewebe ausgeschlossen, so beispielsweise wenn bei Knochentransplantationen der ursprlingliche wirtseigene Knochen samt Periost vollstiindig entfernt wurde. Doch auch in diesen Fällen dürfte nicht eine Umwandlung der bestehenden Strukturen des Transplautates vorliegen, sondern eine nach ursprünglicher Rückbildung von Teilen desselben (des eigentlichen Knochens) vom Transplantat her (vom mitverpflanzten Periost) erfolgende Regeneration mit Herstellung funktionsgemäBer Strukturen. Hier erscheint also tatsächlich eine physiologische Eigenschaft des Transplantates, die Regenerationsart, beeinflußt, allerdings nicht durch eine spezifische Einwirkung des Wirtsorganismus, sondern nur durch eine dem Transplantat durch seine Lage im Wirtsorganismus aufgedrungene, wohl rein passive Funktion. (Widerstand gegen Druck- und Zugwirkungen.)

Wir gelangen nun zu der Besprechung des Gebietes, zu dem auch die vorliegende Arbeit einen bescheidenen Beitrag liefern soll. Es sind hier zweierlei Fälle zu unterscheiden: Solche, bei denen die prinzipielle Entwicklungsart des Transplantates beeinfluBt wird, und solche, bei denen nur der zeitliche Ablauf eines Entwicklungsvorganges am Transplantat durch den Wirtsorganismus bestimmt wird. Zn der ersten Gruppe gehören die außerordentlich interessanten Befunde itber Linsenbildung aus indifferenter Bauchhaut bei Übertragung derselben auf die Augenblase (Lewis 1904, zit. nach Schöne 1912), sowie über Kiemenbildung aus sonst nicht Kiemen bildendem Ektoderm aus der Nachbarschaft der Kiemenregion bei Überpflanzung in die Kiemenregion (EKMaN 1913b). Hier anzuschlieBen wären auch die viel umstrittenen Beeinflussungen transplantierter Gonaden durch den Wirtsorganismus. Diese Frage, die durch ihren Zusammenhang mit der Frage nach der Möglichkeit somatischer Induktion der Keimzellen von gröbter allgemeiner Wichtigkeit erscheint, ist wohl noch als offen zu betrachten. Die positiven Resultate Gurmines (1907) werden vielfach angezweifelt, ebenso führten spätere Versuche zu keinem uberzengenden Befund. In neuester Zeit werden positive Ergebnisse wieder von Kammerer (1913) bei Salamandra maculosa angegeben, jedoch nur bei klinstlich an der Tragamme induzierten Charakteren.

Aus der zweiten Gruppe liegt eine größere Anzahl von Befunden vor. Die Bedeutung dieser Befunde liegt darin, daB durch sie eine Reihe von Fragen, die der deskriptiven Biologie unzugänglich ist, deren Existenzberechtigung sogar erst durch das Experiment bewiesen werden konnte, der Lösbarkeit näher gebracht wird. 
Wenn wir die Entwicklung eines Organismus betrachten, sehen wir, daß immer eine Anzahl ron Prozessen in gesetzmäBiger Weise parallel nebeneinander bergeht, so daß einem bestimmten Stadium des einen Entwicklungsprozesses ein bestimmtes Stadium der andern, sich gleichzeitig am selben Individum abspielenden Prozesse entspricht. Es sind dies erstens die Prozesse, die sich an den in Mehrzahl vorhandenen gleichen Organen abspielen, zweitens aber auch die Entwicklungsprozesse an den verschiedenartigen Organen. Wir können diese Erscheinung, den gesetzmäßig geregelten gleichzeitigen Verlauf von Entwicklungsrorgängen, als Synchronie der Entwicklungsprozesse bezeichnen.

Als Erklärung fur diese Synchronie im normalen Entwicklungsgang der Organismen nahm man fruher wohl allgemein das gleiche Alter der verschiedenen Teile eines Individuums und die gleichen Bedingungen, denen sie ausgesetzt sind, als ausreichend an. Erst das Experiment brachte den Beweis, daß für diese Erscheinungen, sowie uberhaupt fur die Regelung der Entwicklung, noch andere Faktoren maßgebend sind.

Als grundlegend sollen zuerst die Erscheinungen des "Zellsynchronismus « besprochen werden. Gunwirsch (1911) hat darauf aufmerksam gemacht, daß fur die Tatsache, daß sich alle Zellen eines Zellkomplexes meist synchron entwickeln, beispielsweise sich gleichzeitig teilen, nicht allein ihr gleiches Alter und ihre gleiche Vergangenheit verantwortlich gemacht werden können. Auf seine Anregung untersuchte SoroKrna (1912) die Verbältnisse in einem derartigen Falle. Sie stellte zunächst fest, daß normalerweise die zweite Furchungsteilung am Seeigelei in beiden Blastomeren bis auf die feinsten Einzelheiten genall gleichzeitig erfolgt, und ging dann an die experimentelle Untersuchnng dieser Tatsache. Sie trenute die beiden Zweierblastomeren, und zwar entweder durch Schitteln allein oder dureh Zusetzen von Ca-freiem Seewasser und ganz gelindes Schütteln. Die so getrennten Blastomeren blieben - besonders bei Anwendung der zweiten Methode - vollständig ungeschädigt, waren meist nicht deformiert and zeigten normale Entwicklungsgeschwindigkeit im Ablauf der folgenden, zweiten Furchungsteilung. Es zeigte sich aber, daß diese aweite Teilung in den nun unverbunden nebeneinander liegenden Blastomeren in den meisten Füllen nicht mehr genau synchron erfolgte. Da, wie schon bemerkt, eine mechanische Schädigung und damit die Möglichkeit einer Einwirkung verschiedenartiger äuBerer Einflisse auf die beiden getrennten Blastomeren in 
den meisten beriicksichtigten Fällen ansgeschlossen erscheint, dürfte durch dieses Experiment der Beweis dafür erbracht sein, daß normalerweise durch den Kontakt der beiden Blastomeren und wohl einen durch ihn ermöglichten Stoffaustausch eine synchrone Entwicklung der beiden Blastomeren erzielt wird, während nach Aufheben dieses Kontaktes minimale, auch sonst vorhandene, aber durch die gegenseitige Beeinflussung unwirksam gemachte Verschiedenheiten in den beiden Blastomeren verschieden schnelle Entwicklung bedingen.

Hier ist vielleicht auch eine Tatsache anzureihen, auf deren $\mathrm{Zu}$ gehörigkeit zu den Erscheinungen der Synchronie UhцшNнUтн (1913 b) zuerst aufmerksam gemacht hat. HERBst (1912, S. 26) fand, daß Spermakerne von Strongylocentrotus, die in parthenogenetisch sich entwickelnde Eier von Sphaerechinus eindringen, ohne zu kopulieren, in ihrer momentanen Färbbarkeit Schritt halten mit der dem augenblicklichen Teilungszustand entsprechenden Färbbarkeit des Eikernes, bzw. des Kernes derjenigen Blastomere, in die der Spermakern zu liegen kommt.

Aus der weiteren Entwicklung der Organismen sind hauptsächlich einige besonders auffällige Entwicklungserscheinungen daraufhin untersucht, wie weit ihr Verlauf durch den Zusammenhang der Teile des Organismus geregelt ist.

Mit der allgemeinsten Entwicklungserscheinung, dem Altern der Individuen und der dadurch bestimmten Lebensdauer beschäftigte sich Vöchrisc (1892). Er fand, daß bei Pfropfungen die Lebensdauer des Pfropfreises verlängert werden könne, und schrieb diese wie andere Beeinflussungen der Lebensdaner der einen Komponente durch die andere Ernährung'seinftissen zu. Daß die Ernährungseinflitsse die Entwicklungsgeschwindigkeit des Gesamtorganismus beeinflussen können, ist eine bekannte und feststehende Tatsache, ihre spezielle Wirksamkeit bei den Erscheinungen des Alterns ist erst in neuerer Zeit von ChILd (1911) bewiesen und untersucht worden. Es scheint aber - und zwar besonders auf Grund der gleich zu besprechenden Erscheinungen - die Bestimmung der Entwicklungsgeschwindigkeit am Einzelorgan durch andere, von der Ernährung unabhängige Einfliusse vom Gesamtorganismus her zu erfolgen. Zwischen die Beeinflussung des Gesamtorganismus durch die Außenwelt, etwa am Wege der Ernährung, und die Beeinflussung des Einzelorgans durch den Gesamtorganismus ist eine Anderung der Beeinflussungsart eingeschaltet. Wie weit bei den Vöchringsehen Ergeb- 
nissen noch der direkte ErnährungseinfluB eine Rolle spielen mag, und wie weit schon anders geartete Einflisse durch den Organismus, ist eine sehr schwer zu entscheidende Frage.

Bei einer Gruppe von Entwicklungserscheinungen ist uns die Art der Beeinflussung mit groBer Wahrscheinlichkeit bekannt: Bei der Entwicklung der Geschlechtsreife. Hier spielt offenbar bei der Beeinflussung der Organe durch den Gesamtorganismus die innere Sekretion die Hauptrolle. Auf dem Umweg uber sie erst ist die Beeinflussung des Auftretens des geschlechtsreifen Zustandes in letzter Linie auch durch äußere Faktoren, wie Eruährungseinflùsse, möglich. Allerdings können wir dies bisher nur von den Wirbeltieren mit Sicherheit sagen. Wie weit innere Sekretion auch bei den andern Tieren und bei den Pflanzen eine Rolle spielt, ist eine noch offene Frage. Daß aber auch bei Pflanzen der Eintritt der Geschlechtsreife durch Beeinflussung eines Teiles durch einen andern geregelt wird, zeigen wieder Versuche Vöchrings (1892). Er stellte fest, daß das noch mit nicht differenzierten Knospen besetzte Reis der Runkelribe sich zu einem vegetativen Sproßsystem gestaltet, wenn man es mit einer jungen noch wachsenden Wurzel verbindet, daß es dagegen einen Bliutenstand bildet, weun man es im Frühjahr auf eine alte Rübe pflanzt. Hier wird also der Eintritt der Geschlechtsreife der einen Komponente durch den Entwicklungszustand der andern Komponente bestimmt.

Aus dem Gebiet der wirbellosen Tiere liegt iber diese Frage meines Wissens nur ein negativer Befund vor: Koped (1911) 1) fand, daß bei Schmetterlingen die EntwickIungsgeschwindigkeit transplantierter Gonaden durch den Wirtsorganismus nicht beeinflußt wird. Es wäre denkbar, daß dies tatsächlich auf einer prinzipiellen Verschiedenheit zwischen den untersuchten Gruppen besteht, da bei den Schmetterlingen ja auch die sekundären Geschlechtscharaktere im Gegensatz zu den Wirbeltieren durch die Gonaden unbeeinflubbar erscheinen (siehe besonders Mrisenterimer 1907 u. 1908).

Bei den Wirbeltieren liegen mebrere experimentelle Beweise dafür vor, daß hier das Auftreten der für die Geschlechtsreife charakteristischen Erscheinungen vom Gesamtorganismus, und zwar, wie schon gesagt, offenbar durch innere Sekretion vom interstitiellen Gewebe der Gonade her bestimmt werde. Außer den dies beweisen-

1) Bei UHLENHUTH (1913a) ist dieser Befund irrtïmlich in entgegengesetztem Sinne ausgelegt, was ich im Einverständnis mit diesem Autor berichtigen müchte. 
den schon erwihnten Versuchen RIBBERTs sprechen dafur anch die Befunde von Mryss (1912), die besagen; daB das Tempo der Spermatogenese reifer Froschhoden bei Transplantation vom Geschlechtszustand des Empfängers abhängig ist. Unreife Keimzellen werden nicht in diesem Sinne beeinflnßt. Meyss selbst bringt die Befunde mit einer Wirkung der Funktion in Zusammenhang, was mir recht schwer vorstellbar erscheint. Daß unreife Keimzellen unbeeinfluBt bleiben, läßt sich wohl so deuten, daß dem spezifischen Einfluß eben nur bestimmte Stadien überhaupt zugänglich sind.

Eine Beeinflussung eines ganz andern EntwickIungsvorganges stellten ReHN und WaKabayaschi (1912, S. 25-26) fest. Sie fanden, daB bei Knorpeltransplantationen bei jungen Kaninchen der Verknöcherungsprozeß am Transplantat stets genau synchron mit dem an den wirtseigenen Knochen erfolgt. Auch der Verlauf der Verknöcherung muB also vom Gesamtorganismus her geregelt werden.

Einige Arbeiten endlich beschäftigten sich — so wie die vorliegende - mit der Abhängigkeit der metamorphotischen Entwicklungsvorgänge der Amphibien vom Gesamtorganismus. Welcher Art diese Beeinflussungen sind, ist noch unaufgeklärt. Versuche von WiNTREBERT (1905-1911) haben gezeigt, daß das Nervensystem bei dem Ablauf der metamorphotischen Vorgänge im Einzelorgan keine Rolle spielt, ähulich auch Versuche von LoEB (1896). Dagegen ist es bekannt, daß die Gesamtmetamorphose durch äußere Einflüsse wie Nahrung, Wasserhöhe usw. beschleunigt und verzögert werden kann. Aber schon die erste Arbeit, die bei Transplantationen eine Abhängigkeit der Metamorphose der einen Komponente von der der andern bewies, Borss grundlegende * Verwachsungsversuche mit Amphibienlarven (1897) zeigten, daß innerhalb des Organismus der $A b-$ lauf der Metamorphose nicht mehr durch Ernährungseinflìsse geregelt werde (s. Seite 373). Viel eher könnte man zur Erklärung der Regelung der metamorphotischen Erscheinungen innerhalb des Organismus Einfliisse irgendeiner inneren Sekretion heranziehen. Ein experimenteller Beweis hierfür ist aber bisher nicht erbracht. Es ist sogar zu betonen, daß wir für den Ursprung dieses Einflusses noch keinerlei Anhaltspunkte haben. Der Ausdruck: »Beeinflussung durch den Gesamtorganismus s sagt im allgemeinen: Das Organ wird von der Gesamtheit der ubrigen Organe beeinfluBt, ohne daB wir betonen, welches von den iibrigen Organen dabei eine Rolle spielt, auch wenn wir dies - wie etwa bei der Beeinflussung der Sexualcharaktere - wissen. In dem vorliegenden Falle aber könnte der 
Ausdruck vielleicht in einem engeren Sinne zu Recht bestehen. Vielleicht liegt hier gar keine Beeinflussung durch ein spezielles Organsystem vor, sondern tatsächlich ein Einfluß des Gesamtorganismus, ein Einfluß eines allen Teilen gemeinsamen Zustandes, fur dessen Regelung nicht etwa eine von einem Organ ausgehende innere Sekretion, sondern ein allgemeiner Zusammenhang der Organe untereinander sorgt. Daß so etwas denkbar ist, zeigen die Erscheinungen des Zellsynchronismus, besonders die besprochenen Ergebnisse SoroKINAS.

Im einzelnen liegen bisher folgende Befunde vor: BoRN (1897) stellte fest, daß vereinigte Teilstitcke von Anurenlarven sich immer gleichzeitig verwandeln, auch wenn die Ernährungsbedingungen fur die beiden Teile ungleich sind, was sich in verschieden starkem Wachstum und dadurch in verschiedener Größe der Teilsticke äußert. Nur ein Befund veranlaßt ihn zu einer ausnahmsweise gegenteiligen Auslegung, eine vorzeitige Rückbildung eines transplantierten Schwanzes. Doch geht aus seiner eigenen Beschreibung des Falles mit größter Wahrscheinlichkeit hervor, daß in diesem einen Fall gar keine metamorphotische, sondern eine pathologische, degenerative Ruickbildung vorlag. Die Möglichkeit und die große Wichtigkeit der scharfen Unterscheidung zwischen diesen beiden Erscheinungen wird wohl aus meinen Befunden noch deutlicher hervorgehen.

Daß schon fur die von BorN festgestellte Synchronie nicht etwa das gleiche Alter der Teilstucke maßgebend war, geht daraus hervor, daB auch von Tieren desselben Wurfes sich sonst kaum zwei am selben Tag verwandeln, sondern stets außerordentlich große Verschiedenheiten in der Dauer der larvalen Periode auftreten, selbst wenn man mit größtmöglicher Sorgfalt für alle Individuen gleiche Bedingungen zu schaffen sucht.

Speziell mit einer Einzelerscheinung der Metamorphose beschäftigen sich die Untersuchungen UmLenHuTHs (1913a) tiber Augentransplantationen an Amphibienlarven, und zwar hauptsächlich an Salamandra maculosa. In ihnen wurde festgestellt, daß die Verwandlung des transplantierten Auges, ftir die das Verschwinden eines gelben Ringes in der Iris als Kennzeichen aufgestellt wurde, sich unabhängig vom Alter des Spenders zugleich mit der Metamorphose der Augen des Empfänger's vollzieht, also offenbar unter dem Einfluß des Empfängers steht. Nur wenn Spender oder Empfänger zur Zeit der Operation knapp vor der Metamorphose stehen, findet die Beeinflussung nicht mehr statt. Steht der Spender in diesem * Larven- 
endstadium ", dann ist in seinem Körper den einzelnen Organen, beispielsweise den Augen, offenbar der zur Metamorphose nötige Impuls schon erteilt worden und die Verwandlung erfolgt dann am Einzelorgan (Transplantat!) unabhäingig vom Gesamtorganismus (Empfänger!) Ebenso bleibt das Transplantat bei der Metamorphose des Empfängers unverwandelt, weun dieser zur Zeit der Operation schon im "Larvenendstadium" stand, der Spender dagegen noch nicht. Da aber auch hier immerhin eine starke Beschleunigung der Verwandlung des Transplantates eintrat, ist anzunehmen, dab die "Heterochronie s in diesen Fällen darauf beruht, daß.zwischen dem Beginn der Einwirkung des Gesamtorganismus auf das Organ und dem zutagetreten der hervorgerufenen Änderung immer ein gewisser Zeitraum liegen muß.

Mit einer analogen Frage beschäftigt sich unter anderm auch eine Arbeit von WEIGL (1913) tiber Hauttransplantationen an Salamandra macuilosa, Triton und Amblystoma. In bezug auf die Metamorphose fuhrt sie zu folgenden Resultaten: Wenn bei der Transplantation Spender and Empfänger in gleichem Alter standen, so vollzog sich die Metamorphose des Transplantates gleichzeitig mit der des Wirtstieres....

*Wenn bei der Transplantation der Spender jünger war als der Empfänger, so setzte die Metamorphose des Transplantates erst spaiter als die des Wirtstieres ein «...

»Wenn bei der Transplantation der Spender älter war als der Empfäinger, oder wenn der Empfänger abnormal lange im Larvenzustande beharrte, so setzte die Metamorphose des Transplantates frtiher als die des Wirtstieres ein «...

Diese Befunde sind wohl so zu deuten, daß W wendung ungleich alter Tiere stets möglichst große Differenzen wählte - Dr. Uhlenhuth teilte mir mit, daß Dr. Wergu dies auch bei einer Unterredung mit ihm ausdrüklich betonte - und daß daher die sälteren* Tiere wahrscheinlich stets Larvenendstadien waren. Bei dieser Deutung decken sich WErGLS Resultate vollständig mit denen Uhuenhures. Nur der Fall der verfrühten Verwandlung des Transplantates auf einem gleich alten, aber abnorm lang larval bleibenden Empfänger fugt sich dieser Erklärung nicht. Doch ließe sich in diesem Fall wohl nur durch genaue histologische Untersuchung feststellen, ob das Transplantat tatsächlich schon verwandelt war. Wenigstens fuhrten mich meine eigenen Erfahrungen zu der Überzeugung, daß der Prozeß der Hautmetamorphose ein sehr kompli- 
zierter und schwer analysierbarel sei, indem sich bei individuell verschiedenem Verlauf der Gesamtmetamorphose die homologen Stadien oft schwer identifizieren lassen. Auf eine schriftliche Anfrage danach, welche Kriterien WEIGL fur die »Metamorphose * der Haut verwendete, erhielt ich bisher leider noch keine befriedigende Antwort. In seiner Publikation führt WeIGL als Grund für die verspätete Verwandlung die fuir die Beeinflussung zu große Jugend des Spenders, für die verfrübte eine unter Umständen tatsächlich am Transplantat selbständig vorgehende Verwandlung an.

Von besonderem Interesse erscheint mir der weitere Befund Wrigls, daB die vom Wirtstier ausgehende Anregung zur Metamorphose auch über die Grenzen der Art und sogar der Gattung hinaus, also auch bei heteroplastischer Transplantation, wirksam sein kann. Salamanderlarvenhaut auf Tritonlarven überpflanzt, verwandelte sich kurze Zeit nach der Metamorphose des Empfängers in der für Salcmandra typischen Weise. Die geringe Zeitdifferenz läBt sich wohl so erklären, daß der rom Wirtsorganismus zu gleicher Zeit auf die eigene und auf die transplantierte Hant ausgelibte AnstoB zur Metamorphose am artfremden Transplantat sich nur schwerer und langsamer durchsetzen konnte. Noch wichtiger war die Tatsache, daß auch die Haut von Axolotln, auf Salamanderlarven verpflanzt, kurze Zeit nach der Metamorphose des Empfängers in der fưr Amblystoma charakteristischen Art metamorphosierte. Hier erfolgt eine bedeutende Beschleunigung der Metamorphose des Transplantates, da die Axolotl erst viel später und nur dureh besondere äußere Einwirkungen zur Metamorphose hätten gebracht werden können. Die Resultate zeigen, daß der vom Wirtsorganismus ausgehende Einfluß nicht fur die Art spezifisch ist, sondern eine allgemeinere Wirkssamkeit hat. Auch für die Beeinflussung von Sexualcharakteren durch innere Sekretion ist eine derartige Wirksamkeit uber die Grenzen der systematischen Einheit hinaus nachgewiesen worden.

Ich habe die in der Literatur erwähnten Befunde mit größerer Ausfuibrlichkeit und in weiterem Ausmaße besprochen, als es den eigenen Resultaten zu entsprechen schien. Doch hoffe ich, damit Kollegen, die etwa über ein ähnliches Thema arbeiten wollen, einen Dienst erwiesen zu haben, da die betreffenden Angaben teilweise recht zerstreut und sehwer zugänglich sind. 


\section{Fragestellung.}

Die zur Untersuchung gelangende Frage hatte sich aus den UHLenrutuschen Arbeiten ergeben. Es sollte nach den Ergebnissen über die Metamorphose des Amphibienauges ein anderer charakteristischer EntwicklungsprozeB aus dem Komplex metamorphotischer Erscheinungen herausgegriffen und in ähnlicher Weise analysiert werden. Es erschien dabei von vornherein der Prozeß der Kiemenrückbildung als außerordentlich ginstig. Im Verlauf der Untersuchungen ergaben sich allerding's einige technische und methodische Schwierigkeiten, die nicht roranszusehen gewesen waren.

Die zu untersuchenden Fragen lassen sich folgendermaßen präzisieren:

1) Spielt sich an transplantierten Amphibienkiemen ein Vorgang ab, den wir als metamorphotische Kiemenruckbildung auffassen diufen?

2) Zeigt diese metamorphotische Kiemenrickbildung eine Beeinflussung durch den Wirtsorganismus, oder geht sie selbständig, nur von dem Alter and andern Eigenschaften des Spenders abhängig vor sich?

3) Fubrt die eventuelle Beeinflussung durch den Wirtsorganismus auch hier zu einer synchronen Metamorphose, das heißt einer gleichzeitigen Verwandlung des transplantierten Organs mit dem wirtseigenen, unabhängig rom Alter des Transplantates?

4) 'Tritt auch bei Kiementransplantationen bei Verwendung besonders weit entwickelter Larven heterochrone Metamorphose ein?

\section{Die Kiemen der Salamanderlarve und ihre metamorphotische Rïckbildung.}

Die Ruckbildung der Kiemen ist einer der auffallendsten Prozesse bei der Metamorphose der Urodelen. Als Basis einer experimentellen Untersuchung ihres Verlaufes ist eine genaue Kenntnis der dabei sich abspielenden morphologischen, anatomischen und histologischen Einzelvorgänge unumgänglich notwendig. Aber, so merkwîrdig dies klingen mag, eine Beschaffung dieser Kenntnisse aus vorhandener Literatur scheint nicht in ausreichendem Maße möglich zu sein. Vor allem konnte ich in der mir zugänglichen und mir bekannt gewordenen Literatur keine genügenden Angaben iiber die histologischen Prozesse bei der Rückbildung finden, die als Kriterium fiur das Einsetzen der Metamorphose gelten könnten. (Daß solche charakteristisch verlaufende Prozesse vorhanden seien, unterliegt wohl 
keinem Zweifel.) Ferner aber vermisse ich alle genaneren Daten ïber die zeitlichen Verhältnisse der einzelnen metamorphotischen Erscheinungen and über die Grenzen ihres Schwankens unter natirlichen Umstiuden. Soweit es mir die Zeit gestattete, habe ich diese Lücken durch eigene Untersuchungen zu füllen gesucht, doch muß ich gleich gestehen, daß dies bisher leider nicht sehr weit war.

Nähere Beschreibungen der änßeren Kiemen der Amphibienlarven finden sich vor allem bei Clemens (1895) und OPPeL (1905),

Fig. 1.

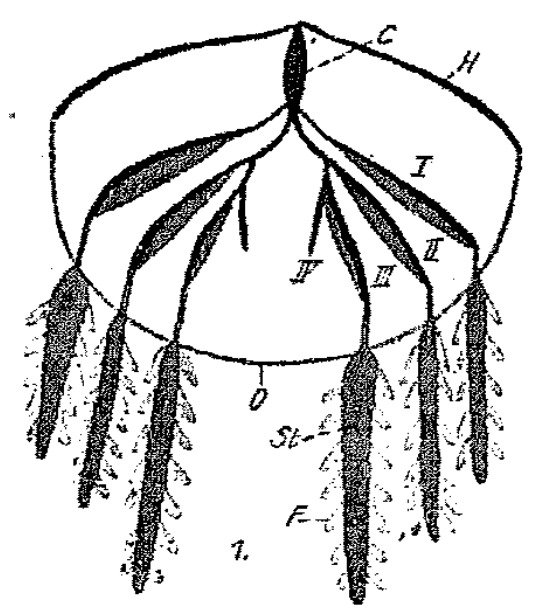

Fig. 3.

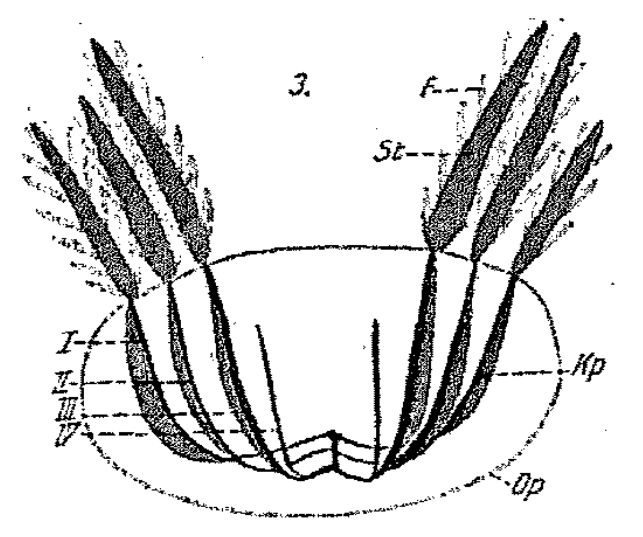

Fig. 2.

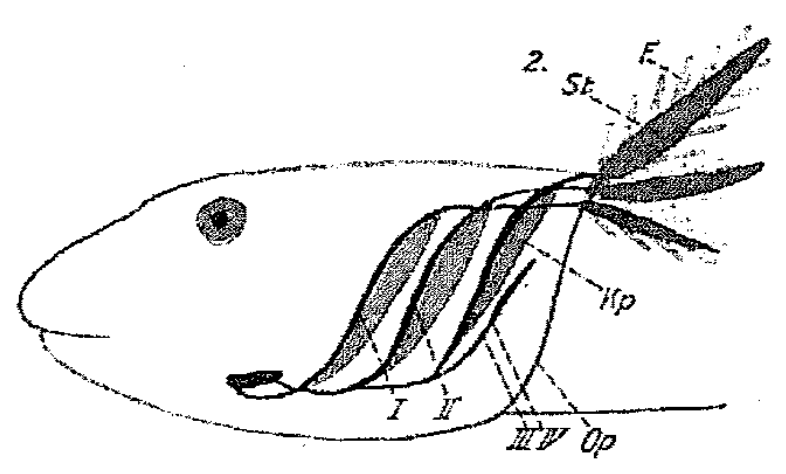

Fig. 1-3. Suhomatisohe Darstellung des Kiemenapparates der Urodelentarven.

$I, I T, I I I, I Y$ 1.-4. Kiemenbogen, $K_{p}$ Kiemenplittehen, St Kiemengtímmo, $I F$ Kiomonflodern, $H$ Hyoidbogen, $O$ Operenlarfalte, $O$ Copula.

1. Projektion auf dio Horizontalobene,

2 Projeltion anf dio Sagittalobone,

3 Projektion auf dio Transversnlobene.

einzelne wichtigere Angaben besonders bei BoAs (1882 und 1883) und MAURer (1888a und 1888b).

Der Kiemenapparat der Larven ron Salamandra maculosa (Textfigur 1-3) besteht hauptsächlich aus vier Teilen: Den Kiemenbögen, den Kiemenplättchen, den Kiemenstämmen oder Kiemenkörpern und den Kiemenfransen, Kiemenfäden oder Kiemenfiedern. Die beiden letzteren Teile bilden die eigentlichen äußeren Kiemenanbänge. Die Kiemenbogen ziehen von der vorn unten median liegenden Copula nach rỉckwärts oben lateral, wo sie frei unter dem Integument enden, jederseits in Vierzahl und in verschiedener Länge. Nach rückwärts 
hängen die Kiemenplättchen von ihnen herab, dünne Hautlamellen mit ein- bis zweischichtigem Epithel, spärlichem Bindegewebe und relativ reichen Blutgefäßen, offenbar auch mit respiratorischer Funktion. Dieser ganze Komplex ist durch eine vom Hyoidbogen ausgehende ventrale Hautfalte, die Opercularfalte, umwachsen.

An den ruckwärtigen (also oberen und lateralen) freien Enden der drei ersten Kiemenbogen setzen die Kiemenstämme an, an der Stelle, wo die Opercularfalte die dorsale Körperwand erreicht. Ihre Dorsalfäche ist breit und gewölbt und erscheint als direkte Fortsetzung der Körperwand, mit der sie auch teilweise gleichen histologischen Bau aufweist. Von den seitlichen unteren Kanten der Stämme gehen beiderseits die Fiedern aus. Form und Größenverhältnisse von Stämmen und Fiedern schwanken auBerordentlich. Nach der Geburt, besonders aber bei ktinstlicher Entnahme aus dem Uterus, werden die noch an die Atmung im Uterus angepaßten besonders langen und schlanken Fiedern kürzer und plumper. Dann tritt, parallel gehend mit dem Wachstum der ganzen Larve, wieder ein Wachstum der Kiemen mit Vermehrung der Fiedern ein, das bis kurz vor der Metamorphose anhalten kann. Daneben kommen aber noch individuell außerordentlich wechselnde Form- und GröBenveränderungen vor.

Das histologische Bild der äuBeren Kiemen (siehe die Fig. 9-13) ist ein ziemlich einfaches. Ein Teil der dorsalen Fläche der Kiemenstämme ist von einem dem normalen Körperepithel sehr ähnlichen und als direkte Fortsetzung desselben erscheinenden Epithel bedeckt. Es besteht hauptsächlich aus einer basalen und einer distalen Schicht von kleinen, plasmaarmen Deckzellen und einer zwischen diesen beiden liegenden fast kontinuierlichen nur streckenweise zurtucktretenden Schicht Lexdrgscher Zellen, der bekannten großen, plasmareichen Drüsenzellen mit körnig erscheinendem Sekret and kleinen meist runden Kernen. Die Kerne der Deckzellen sind verhältnismäßig groß, meist länglich, aber von sehr wechselnden Umrissen, die sich anscheinend ganz der wechselnden Form der Zelle anpassen. Gegen das die Kiemenstämme und Fiedern füllende Bindegewebe erscheint dieses Epithel durch eine starke, faserige Basallamelle, nach außen durch einen mittelstarken, in den Präparaten gekörnelt erscheinenden Grenzsaum abgeschlossen. An der Ansatzstelle der Kiemenstämme liegen unter diesem Epithel außerordentlich große Giftdrussen, außerdem Schleimdrüsen und indifferente Drüsenanlagen in den verschiedensten Entwicklungsstadien. Diese Drỉsen weisen hier den- 
selben Bau auf, wie in der übrigen Körperhaut. Die Giftdrüsen zeigen hier besondere Größe und Häufung und gehen an dieser Stelle teilweise, bei der Metamorphose wohl direkt, in die riesigen Drusen des Parotidenfeldes tiber. Die sonst itber den Körper verstreuten Giftdrüsen sind spärlicher und viel kleiner, nur zu beiden Seiten der dorsalen Mittellinie erreichen sie ähnliche Dimensionen wie an der Ansatzstelle der Kiemenstämme.

Ein ganz anderes, mit dem eben besprochenen allerdings durch kontinuierliche Übergänge verbundenes Bild bieten die übrigen Teile des Kiemenepithels, die Epithelien der Fiedern, der ventralen Fläche und des distalen Teiles der dorsalen Fläche der Kiemenstämme. Hier sind keine oder pur vereinzelte LEYDIGsche Zellen vorhanden, Drtusen fehlen fast ganz und das Epithel besteht je nach der proximalen Lage des Teiles und der GröBe der ganzen Kieme aus einer bis etwa sieben Schichten von großkernigen Deckzellen, die wohl auch plasmaarm sind, aber doch einen etwas beträchtlicheren Zellleib aufweisen als die Deckzellen des ubrigen Körperepithels. Ihre Gestalt ist kubisch bis plattenförmig. Die Basallamelle fehlt hier, offenbar damit jede die Atmungsprozesse hindelnde Trennung zwischen dem die Kiemen umspulenden Wasser und den subepithelial liegenden Blutgefäßen möglichst vermieden werde.

Das die Stämme und Fiedern erfüllende Bindegewebe zeigt faserige Strukturen. Die Gefäße liegen in den Fiedern dicht subepithelial, oft sogar sich stellenweise zwischen die basalen Teile der Epithelzellen einzwängend. Im Stamme liegt die Arterie ventral, vom Bindegewebe umhillt, die Vene dorsal nahe dem Epithel. Beide sind meist ron Pigment begleitet, das sich aber auch sonst im Bindegewebe, im Epithel der Stämme, und stellenweise auch im Epithel der Fiedern findet. Die Unterscheidung von Arterien und Venen, die mir später oft sehr wichtig wurde, ist nicht immer leicht, gelingt aber oft ganz gut nach dem Verhältnis der Dicke der Gefäßwand zum Lumen und nach dem Aussehen der Intima.

In den Stämmen liegt ferner eine recht kräftige Muskulatur und nahe dem Ende der Kiemenbogen, knapp an der Ansatzstelle also, die Thymus (Fig. 11).

Die Kiemen bleiben meist bis zu den späteren Stadien der Metamorphose erhalten. Dann setzt erst eine langsame, meist unbedeutende Rückbildung ein, und erst in den letzten 1-3 Tagen des Wasserlebens erfolgt dann eine rapide Rückbildung des weitaus größten 
Teiles der gesamten äuBeren Kiemenanhänge. Seltener sieht man schon längere Zeit vorber eine bedeutendere Rückbildung beginnen. Tiere, bei denen das der Fall ist, zeigen meist auch andere Unregelmäßigkeiten in ihrer Metamorphose, so beispielweise abnorm langes Verweilen in einem Stadium, indem das Tier bald im Wasser, bald am Land ist, ein Stadium, das sonst meist, wenn es überhaupt auftritt, kaum einen Tag dauert. Nach der dem ans Landsteigen unmittelbar vorausg'ehenden rapiden Rückbildung des größten 'Teiles der änßeren Kiemenanhänge sind äußerlich meist als Kiemenreste noch kleine Warzen oder Zacken zu sehen, die dann erst langsamer im Laufe der folgenden 2-14 Tag'e vollständig verschwinden.

Zur selben Zeit wie die hauptsächliche Kiemenrùckbildung, also meist in den letzten 1-3 Tagen des Wasserlebens, erfolgt fast immer auch eine plötzliche Beschleunigung in der Ritckbildung des Rudersaumes am Schwanze. Doch handelt es sich hier noch deutlicher nur um eine Tempoänderung in einem schon vorher begonnenen und. beim ans Landgehen meist noch nicht ganz vollendeten Prozeß. Denn auch am Rudersaum beginnt schon einige Zeit vor der rapiden Ruckbildung des gröBten Teiles eine langsame Rückbildnng kleinerer Stucke und anch rom Rudersaum sind auch nach dem ans Landgehen, also nach der rapiden Rutckbildung des größten Teiles von Kiemen und Rudersaum, oft noch kleine Reste vorhanden, die erst wieder langsamer vollständig versohwinden.

Während die Tiere noch am letzten Tage vor dem ans Landgehen oft lebhaft nach Nahrung schnappen, werden sie nach dem ans Landgehen auffallend träge und nehmen längere Zeit keine Nahrung zu sich.

Mit den zeitlich, wie es scheint, stets eng verbundenen drei Vorgängen: Rapide Riickbildung des größten Teiles der Kiemen und des Ruderschwanzes und ans Landsteigen fand ich noch einen vierten Vorgang, wie ich glaube, stets zeitlich eng verknüpft: eine vollständige Häntnng, die ebenfalls fast immer am letzten Tage vor dem ans Landsteigen erfolgt. Doch glaube ich, daß die vier Vorgäng'e nicht etwa in der Weise verbunden sind, daß bei dieser Häutung: der größte Teil der Kiemen und des Rudersaumes einfach mit abgeworfen werden. Denn ich glaube mit Sicherheit konstatieren zu können, daß wenigstens stundenlange Differenzen zwischen der Häutung und den Rickbildungsprozessen vorkommen, daß besonders die Kiemenriackbildung meist noch vor der Häutung erfolgt.

Während diese Zusammenhänge mir fur die überaus wichtige 
Beurteilung des Stadiums, in dem sich ein Versuchstier momentan befindet, wertvolle Dienste leisteten, fand ich andere metamorphotische Erscheinungen, wie die sIrispigmentierung* und besonders auch die verschiedenen Farbänderungsschritte in weitestem Ausmaße unabhängig von dem besprochenen Erscheinungskomplex. Eine Untersuchung dieser Verhältnisse, besonders bei Heranziehung anch del den Prozessen zugrunde liegenden histologischen Veränderungen, würde wohl noch manches wichtige Resultat bringen und sowohl der deskriptiven als auch der experimentellen Biologie wertvolle Anregungen bieten.

\section{Arbeitsmethode.}

In der Operationstechnik konnte ich mich fast vollständig an die der UHt.menthschen Augentransplantationen anschließen. Es wurden Larven von Salamandra maculosa in den verschiedensten Altersstufen verwendet. In der I. Serie, die hauptsächlich technisch orientierende Vorversuche enthielt, wurden Larven, die Anfang September dem Uterus der Mutter entnommen worden waren, nach $3-4$ Wochen operiert; in der II. Serie solche, die am 21. Oktober dem Uterus entnommen worden waren, nach 8-10 Wochen. Diese Serie sollte besonders einen etwaigen Einfluß eines Abschneidens der eigenen und der transplantierten Kiemen behandeln, fuhrte aber in dieser Beziehung: zu keinem endgultigen Resultat. Die Tiere beider Serien zeigten bei der Operation noch nur früh-larvale Charaktere. Von ihnen verwandelten sich die ersten - dabei sind sowohl die operierten als auch die Kontrolltiere bericksichtigt - 6 Monate nach der Entnahme, also 5 bzw. 4 Monate nach der Operationszeit, anderseits waren 9 bzw. $71 / 2$ Monate nach den Operationen einzelne Tiere noch larval. Die III. Serie diente der Hauptfrage: dem Verhalten des Transplantates bei Verwendung verschieden alter Larven. Hier wurden drei verschiedene Altersklassen verwendet: 1) Larven, die 5-6 Monate vor der Operation dem Uterus entnommen worden waren und ron denen sich die ersten etwa zur Zeit der Operationen (17. III. bis 29. IV.) verwandelten, die letzten etwa $2 \frac{1}{2}$ Monate nach den Operationen. 2) Larven, die 2-7 Wochen nach der Uterusentuahme zur Operation gelangten (17. III.-29. IV.). Von diesen verwandelten sich die ersten etwa einen Monat nach der Zeit der Operationen, viele waren bei Abbruch der betreffenden Versuche $(21 / 2-4$ Monate nach der Operation) noch larval. 3) Larven, die 10-15 Tage vor den zwischen dem 24. und 29. IV. stattfindenden Operationen ge- 
worfen worden wiren. Von diesem Wurf verwandelte sich die erste Larve $2 \frac{2}{2}$ Monate nach der Operation (siehe Tab. I).

Die angegebenen Daten, sowie auch die einzelnen Notizen der Protokolle zeigen, daß - was ja zu erwarten ist, aber in der Literatur manchmal bestritten wird, - das Herbstmaterial sich im Durchschnitt viel langsamer entwickelt als das Frühjahrsmaterial. Auch erscheint es weniger widerstandsfähig als dieses. Vom Frühjahrsmaterial wieder verwandelten sich die Anfang März dem Uterus entnommenen Larven schneller als die im April geworfenen. Ob auch hierin eine GesetzmäBigkeit liegt, oder ob es nur Zufall war, kann ich bei dem fur solche Zwecke zu geringen mir zur Verfugung stehenden Material nicht entscheiden.

Bei der Operation wurden stets rechte und linke Kiemen eines Tieres (Spenders) auf zwei verschiedene Wirtstiere (Empfanger) übertragen. Alle drei Tiere wurden mit Ätherdampf narkotisiert. Der Spender wurde im Wasser operiert, da man außerhalb des Wassers die sich dann glatt an den Körper anlegenden und dadurch schlecht sichtbaren Kiemen leicht verletzt. Die Empfänger wurden auf feuchtem Filtrierpapier operiert, was den Vorteil hatte, daß man sie nicht so stark narkotisieren mußte (da die Tiere im Wasser schneller aus der Narkose erwachen, als in der Luft) und sie so die Schäden der Narkose leichter iuberstanden. Nur selten geschah es, daß trotzdem ein Empfänger den Folgen einer zu starken Narkose in Verbindung: mit denen der Operation erlag. Bei den Versuchen der II. Serie machte ich die Beobachtung, daß Tiere mit gestutzten eigenen Kiemen die Narkose viel schwerer vertragen, als solche mit unverletzten Kiemen. Ob das darauf zurtickzufuhren sei, daß die Schwächung: durch das Stutzen die Tiere überhaupt weniger widerstandsfähig mache, oder ob das darauf beruhe, daß bei der Erholung aus der Narkose die Kiemenatmung eine wichtige Rolle spielt, kann ich noch nicht entscheiden, doch halte ich das letztere aus mehreren Grinden fur wahrscheinlicher. - Der Spender, der nicht weiter beobachtet werden sollte, ging meist noch während der Operation an den Folgen des Eingriffes, der ihn viel stärker schädigt als den Empfänger, ein.

Die Kiemen wurden mit einem ziemlich großen Stuck der vor ihnen liegenden Haut (Fig. 1 u. 3) und den darunter liegenden Teilen: Bindegewebe, Muskulatur, Teile der Kiemenbogen und der Kiemenplättchen usw. abgetragen. Dann wurde das Transplantat noch unter Wasser von allzn großen anhaftenden Gewebefetzen befreit und nach 
Form und Größe einer schon vorher in der Nackengegend des Empfängers durch Abtragung von Haut und Rückenrumpfmuskulatur geschaffenen $W$ unde zugesehnitten. Fur diese Wunde wurde nach verschiedenen, orientierenden Vorversuchen stets die Nackengegend gewählt. Sie bot folgende Vorteile: Sie gestattet in den weitesten Grenzen die Wunde beliebig klein oder groß zu machen, ohne daß man dabei auf prinzipiell verschiedenen Untergrund stößt. Die hohe Muskelschicht an dieser Stelle ermöglicht eine starke Aushöhlung: der Wunde, ohne besondere Schädigung des Empfüngers, was das Verwachsen des Transplantates meist sehr fördert. Endlich ist diese Stelle auch bei lobhaftester Bewegung des Tieres Anstößen kaum ausgesetzt und auch durch die Krümmungen des Körpers selbst wird sie kaum in Mitleidenschaft gezogen. Man darf daher dort auf ein ziemlich ungestörtes Anheilen hoffen. Da die ganze Haut der Lavre respiratorisch tätig ist, duirfte auch an dieser Stelle die Blutversorgung: - ein, wie später noeh näher gezeigt werden soll, äußerst wichtiger Faktor - nicht ungunstig sein.

Beim Übertragen des Transplantates muBte besonders durch kleine Kunstgriffe dafur gesorgt werden, daß der weiche Hautlappen sich nicht zusammenrollt oder faltet, was besonders beim Herausnehmen aus dem Wasser leicht geschieht. Das Transplantat wurde stets so aufgelegt, daß die frei abstehenden Kiemenstämme nach rückwärts gerichtet waren, so daß sie bei der Vorwärtsbewegung des Tieres wie an ihrem normalen Sitz nicht aufgehoben, sondern an den Körper angedruckt werden. Bei der Operation wurde zur Desinfektion der Wunde, der Instrumente und der Gefäße eine hellrosa Lösung von Kaliumpermanganat verwendet.

Nach der Operation blieben die Tiere 24 Stunden in einer feuchten Kammer. In dieser Zeit, meist schon nach wenigen Stunden, ist das Transplantat so fest mit der Unterlage verwachsen, daß die Tiere wieder ins Wasser gebracht werden konnten. Sie wurden nun einzeln in 3 Liter fassenden Einsiedegläsern gehalten, die bis $1 / 3$ ihrer Höhe mit Wasser gefüllt und mit Glasplatten zugedeckt wurden. In der ersten Woche nach der Operation wurde das Wasser täglich, später etwa alle 3-7 Tag'e g'ewechselt. Jedes Tier bekam gewöhmlich jeden 2. oder 3. Tag eine kleine Portion Tubifex. Bei älteren Larven wurde durch Beifitigen von Kies, der an einer Stelle den Wasserspiegel iuberragte, Gelegenheit zum Verlassen des Wassers gegeben. Wann dies geschah, und in kritischen Zeiten auch wann gefuttert wurde, wurde bei jedem Tier, bzw. bei jeder Gruppe gleich- 
behandelter Tiere, notiert, damit erentuelle Beeinflussungen der Metamorphose konstatiert werden konnten. Jedes Versuchstier wurde in kritischen Zeiten tiiglich, sonst meist alle 2-5 Tage angesehen und die Beobachtungen möglichst genan und objektiv ohne Berticksichtigung der vorhergehenden notiert. Dadurch kamen allerdings in die Protokolle manchmal, besonders bei Beschreibungen quantitativer Verhältnisse, kleine Widersprtiche, die sich aus der Schwierigkeit der Beurteilung ergeben.

Von allen charakteristischen Stadien wurden erstens Belegexemplare in Formol konserviert (nach solchen sind auch die Fig. 4, $5,6,7,8$ angefertigt) und zweitens Transplantate samt ihrer Umgebung anatomisch und histologisch untersucht. Die Untersuchung: galt vor allem den morphologischen Transplantationsresultaten, ferner aber besonders den histologischen degenerativen und metamorphotischen Veränderungen. Gerade dieser histologische Teil der Arbeit ist als der noch unvollendetste zu bezeichnen.

Zur Fixierung wurde vor allem die Mischung KaliumbichromatFormol-Eisessig (7:2:1) und Dreifachfurbung mit Delafieloschem Hämatoxylin-Säurefuchsin-Orangealkohol verwendet. Sehr reichliches mit Sublimat-Eisessig, mit FLemmosscher und mit Znewenscher Flussigkeit behandeltes Material konnte bisher noch nicht zux Untersuchung gelangen.

Zweigversuche tuber die Wirkung des Haltens im feuchten Raum statt im Wasser auf normale und transplantierte Kiemen wurden besonders in einer eigenen, IV. Versuchsserie begonnen, fuhrten aber bisher zu keinem beweisenden Resultat.

\section{Ergebnisse der Transplantationen.}

Die Resultate der vier Serien, in die die experimentelle Untersuchung zerfiel, sollen hier gemeinsam besprochen werden.

Das direkte Operationsresultat ist aus den Abb. 3, 4, 14, 15, 16 zu ersehen, die Transplantate kurz nach der Operation darstellen. Sie zeigen die schon im Abschnitt "Arbeitsmethode " angegebenen Bestandteile: Bei äußerer Betrachtung Kiemenstämme und Fiedern und die davorliegende mittibertragene Haut, anf Schnitten außerdem die inneren Teile, Reste der Kiemenbogenknorpel, Thymus, Bindegewebe, Muskulatur und Blutgefäße.

Nach der Operation setzt am Transplantat eine langsame Ruckbildung der Kiemenfiedern und teilweise auch der Kiemenstämme 
ein. Diese Rückbildung geht sehr verschieden weit und erreicht nach etwa 4-6 Wochen meist ihren Hobepunkt. Nach dieser Zeit oder auch schon etwas fruber tritt ein Stillstand und in manchen Fällen wahrscheinlich auch eine gering'e Wiederaufdifferenzierung ein. Im allgemeinen kann man von dieser Zeit an ein deutliches Dauerstadium unterscheiden, währenddessen das Transplantat sich im wesentlichen unverändert erhält. Es bietet in diesem Stadium meist folgendes im einzelnen allerdings anßerordentlich variables Bild: In der Nackengregend erhebt sich ein Höcker, dessen Umriß etwa ellipsenförmig ist. Seine Länge beträgt meist $2-7 \mathrm{~mm}$, seine Breite $2-5 \mathrm{~mm}$, seine Höhe $1 / 3$ bis $1 \frac{1}{2} \mathrm{~mm}$. Die Färbung seiner Haut weicht meist von der der Umgebung $a b$ und die Unterbrechung der Zeichnung der wirtseigenen Haut läßt sich fast immer unverändert erkennen. Am Hinterrande des Höckers stehen drei verschieden lange Kiemenstämme frei $a b$, die noch Reste der Fiedern in Form kurzer Fäden oder verschieden geformter Zacken tragen (siehe die Fig. 5, 6, 7).

Die anatomische Untersuchung zeigt folgende Verhältnisse: In dem Höcker liegen unter normal aussehendem Integument die mitübertrag'enen Teile der Kiemenbögen, deren Knorpel gut erhalten erscheint, and die Thymus, die ebenfalls kanm irgendwelche pathologische Verinderungen erkennen $1 \ddot{a} B t$, eingehtult in Bindegewebe, das von ziemlich starken oft mit Blutkörperchen reich geftulten Gefäßen durchzogen wird (Fig. 17). Nach unten geht das Bindegewebe des Transplantates in das die Muskulatur umhlullende Bindegewebe des Wirtes direkt uber.

Die Blutgefäße lassen sich aus dem Transplantat noch ziemlich weit in das Gewebe des Wirtstieres hinein leicht verfolgen (Fig. 18), doch war es mir bisher nicht möglich, zu sehen, von welchen Hauptgefäßstimmen des Wirtes sie stammen. Sie scheinen meist durch den Bindegewebsstreifen zu ziehen, der die beiden Sèiten des Rückenrumpfmuskels trennt. Manche von ihnen scheinen im Höcker selbst blind zu enden, andere ziehen in die Stämo und offenbar auch in die Fiedern, da auch in diesen gefullte Blutgefäße zu sehen sind (Fig. 19 u. 20).

Die vom Körper abstehenden Stämme und Fiederreste zeigen ebenso wie die im Höcker liegenden Teile des Transplantates gröBtenteils ein gesundes, normales Aussehen. Blutgefäße lassen sich fast ebenso zahlreich wie in normalen Kiemen nachweisen, nur scheinen sie nicht so glatt und regelmäBig zu verlaufen wie dort.' Auch trifft man, besonders in den Fiederresten, neben normal aussehenden gefullten Gefaßßn (Fig. 20), leere, die einen degenerierten Eindruck 
machen (Fig. 21). Die Bilder scheinen mir tiberhaupt zu besagen, daß - wenigstens in den peripheren Teilen - die ursprtinglichen Gefäße verölen und eine vollständige Neuvaskularisierung vom Wirtsorganismus her erfolgt. In den Fiedern scheinen die neuen Gefäße noch nicht so dicht an das Epithel angeschmiegt zu liegen, wie normale KiemenfiedergefäBe (Fig. 20 u. 13).

Die Epithelien zeigen die für die betreffenden Teile charakteristischen Eigenschaften. Höchstens wäre es möglich, daß die LEYDIGschen Zellen in dem dorsalen Kiemenstammepithel abnorm stark zurücktreten. Die großen Giftdrüsen sind vollständig erhalten und zeigen verschiedenste Stadien der Sekretproduktion. Im Epithel des Transplantates finden sich auch Mitosen, stellenweise sogar in beträchtlicher Hänfigkeit, was auch fur ein normales Weiterleben der Gewebe spricht (Fig. 19).

DaB eine morphologische Wiederaufdifferenzierung der transplantierten Kiemen nicht oder nur in außerordentlich geringem Maße stattfindet, schien mir im Anfang tuberraschend. Denn bei UHLENHUTHs Augentransplantationen trat wohl auch anfänglich eine Rückdifferenzierung: der feineren histologischen Elemente ein, nach kurzer Zeit aber erfolgte eine völlige Wiederherstellung der normalen anatomischen und histologischen Verhältnisse. Wenn bei einem so komplizierten Organ, das an seine Umgebung doch anscheimend viel gröBere Anspruche stellt als die viel einfacher erscheinenden Kiemen, bei Versetzung an einen g'ewissermaßen naturwidrigen Ort eine Wiederanfdifferenzierung erfolgt, dann mißte man doch annehmen, daB bei den Kiemen dasselbe der Fall sein wirde, besonders, da die schon erwähnten Tatsachen beweisen, daB das Transplantat am neuen Ort am Leben erhalten bleibt und auch seine Eigenart bewahrt, daB die Operationen also als gelungen anzusehen sind.

Gegen diese Argumentation aber erhob sich bald ein Verdacht. Es ist nämlich denkbar, daß die Kiemen ihrer Funktion entsprechend an ihre Umgebung doch größere Anspriche stellen als die morphologisch und anatomisch so viel komplizierteren Augen. Roux stellte fur die vollständige Erhaltbarkeit eines Transplantates zwei Hauptbedingungen auf: Vorhandensein funktioneller Reize und gentugende Ernährung. Die Notwendigkeit der ersten Bedingung wird neuerdings vielfach angezweifelt. Bei den transplantierten Augen ist ein Funktionieren wohl ausgeschlossen und auch die einfache Einwirkung des Lichtreizes scheint nach anf dem Naturforschertag 1913 mitgeteilten neneren Ergebnissen UnLenнuтнs keinen Einfluß auf das 
Verhalten des Transplantates zu haben. Dagegen beweisen alle Versuche uber Transplantationen die außerordentliche Wichtigkeit der Ernährung des Transplantates. Man könnte sich nun vorstellen, daß die Kiemen am normalen Ort infolge der funktionellen, außergewöhnlich reichen Blutzufuhr anch an die Verwendungsmöglichkeit exzeptionell großer Blutmengen furr ihre Ernährung gewöhnt sind, wie sie ihnen nach der Transplantation nicht im selben Ausmaße zur Verftugung stehen. (Auch die Qualität des Blutes könnte in diesem Falle eine Rolle spielen.)

Eine Bestätigung für die Wichtigkeit der charakteristischen Blutzufuhr ftir die morphologische Entwicklung der Kiemen liegt in ei- nigen Resultaten der schon zitierten Arbeit Ersians (1913a), der an Anurenembryonen bei Transplantation des Ektoderms, das normalerweise die äußeren Kiemen liefert, vor Beginn der Kiemenbildung, wohl eine Kiemenbildung beginnen sab, aber feststellen mußte, daß die gebildeten Kiemenanlagen keine Blutzirkulation erhielten und bald resorbiert wurden. Er führt dies auf das Fehlen der Kiemenarterie zurtick; denn wenn das betreffende Ektoderm in der noch den Kiemengefäßen zugänglichen Region - wenn auch in abnormer Lage - aufgesetzt wird, dann tritt Blutzirkulation und normale Weiterentwicklang von abnorm gelagerten Kiemen ein. EKMAN betrachtet daher das Kiemengefäß als notwendigen $*$ Ausfulurungsfaktor * fur die Entwicklung der äußeren Kiemen.

Es lag nun die Befürchtung nahe, daß das Transplantat, das infolge der ungünstigen Verhältnisse einer Aufdifferenzierung nicht fähig war, auch die metamorphotischen Erscheinungen nicht mit der notwendigen Deutlichkeit zeigen whirde. Diese Befürchtung erwies sich aber als ungerechtfertigt.

Das Dauerstadium, bzw. die ihm vorangehende langsame, kontinuierliche, an die Operation direkt anschließende teilweise Rulckbildung am Transplantat bielt so lange an, bis das Wirtstier in jenes kritische Stadium der Metamorphose trat, das dadurch kenntlich war, daß die schon erwähnten Vorgänge erfolgten: Häutung, besonders starke Beschleunigung in der Rickbildung der wirtseigenen Kiemen und des Rudersanmes, und in den hierauf folgenden 1-3 Tagen ans Landsteigen des Tieres. In diesem Stadium trat auch eine plötzliche Rutckbildung fast all dessen ein, was eben noch von den Stämmen und Fiedern der tibertragenen Kiemen je nach dem momentanen Riickenbildungs- oder Dauerstadium des Transplantates vorhanden war. Daß hier ein anderer Vorgang vorlag als die anfängliche Rück- 
bildung am Transplantat, beweist eine Reihe von Tatsachen. Bevor ich aber diese zusammenfassend bespreche, möchte ich fur die Trennbarkeit der beiden Vorgäinge einige Beispiele bringen. Ich gebe hierzu einige Notizen aus den Protokollen wieder, und zwar vorderhand ohne jede Bertucksichtigung des Verhaltens des Wirtstieres, damit das Verhalten des Transplantates um so klarer hervortrete.

Dem Protokoll des Tieres I, $12=\mathrm{I}$. Versuchsserie, 12. Versuch) entnehme ich folgende Daten:

$$
\text { ^Operation am 1. X. 1912." }
$$

*7. XII.: Transplantat zweistïmmig mit kurzen dicken Fiedern, davor hautbedeckte Aufwölbung. . *

$» 20$. III. 1913. Transplantat zwei schwach 'gezackte Stämme (hinter der Aufwölbung) ...

*30. III. Transplantat als Aufwölbung. .*

Zwei Kiemenstämme hatten sich also ỉber $5 \frac{1}{2}$ Monate erhalten, als Fiederreste waren bis zuletzt schwache Zacken zu sehen. Und nun erfolgt plötzlich, in einem Zeitraum von längstens 10 Tagen, eine plötzliche, vollständige Ruickbildung der Kiemenstämme.

\section{II, 2. Operation 24. XII. 1912.}

*14. I. 1913. Alle drei Stämme gut erhalten, Enden schwarz, mit kleinen weißen Fiedern ...

*10. III. Drei kurze gesprenkelte, ausgezackte Stämme..*

»31. III. Drei schöne Stämme ohne Fiedern ..

»23. IV. Drei große Stämme mit wenigen kleinen Zacken ..

*1. V. Transplantat drei schöne, große Stämme...

*6. V. Transplantat drei deutliche, mittellange Stämme...

*8. V. Transplantat Aufwölbung mit kurzen Stummeln ...

*10. V. Transplantat nur mehr schwache Aufwölbung..."

Hier hatten sich alle drei Stämme tuber 4 Monate gut erhalten, dann setzte plötzlich eine sehr rasche, aber doch noch über mehrere Stadien verfolgbare vollständige Ruickbildung ein.

II, 4. Operation am 19. XII. 1912.

×10. III. 1913. Transplantat kurze Stämme mit weißem, gezacktem Saume... Saum ...*

*17. IY. Transplantat drei kurze, gezackte Stämme mit weißem

„23. IV. Freier Teil des Transplantates groB, dreilappig... 
Abhängigkeit der metamorphotischen Kiemenrïckbildung nsw.

Nun folgen nur Notizen uber das Gleichbleiben des Transplantates, so noch am 5. V. eine ausdrickliche Bemerkung daribber. Am 6. V. steht tiber das Transplantat die Bemerkung: ${ }^{\text {Aufwölbung mit }}$ ganz kurzen StummeIn. « Am 8. V.: *Transplantat nur mehr kleine Aufwölbung. * Hier war also die plötzliche Rückbildung des größten Teiles innerhalb von 24 stunden erfolgt, nachdem sich ansehnliche Stammreste $41 / 2$ Monate erhalten hatten.

\section{II, 6. Operation am 19. XII. 1912.}

^17. IV. 1913. Am Transplantat drei schöne glatte Stämme..* »23. IV. Transplantat innverändert. .*

»26. IV. nnverändert (!) . .

1. V. Transplantat nur als Anfwölbung. *

Nachdem sich drei schöne Stämme über 4 Monate erhalten hatten, plötzlich vollständige Rückbildung in längstens 4 Tagen.

III, 9. Operation am 5. IV. 1913.

*8. IV. Drei verschieden große Stämme mit Fiedern ...

*12. IV. Am Transplantat drei gefiederte Stämme...

»16. IV. Transplantat mit Fiedern ...

- 23. IV. Fiedern noch deutlich, aber kurz...

*25. IV. Am Transplantat noch deutliche Zacken ..*

^26. IV. Am Transplantat deutliche Zacken ...

*29. IV. Am Transplantat deutlich schwach gezackte Stämme . .*

$\gg$ 1. V. unverandert...

»3. V. unverändert . .*

๑5. V. Am Transplantat drei deutlich gezackte Stämme..*

*6. V. Transplantat schwach gezackt ..*

$\gg 9$. V. unverändert. .*

»10. V. Am Transplantat drei schöne, ganz schwach ausgezackte Stämme...

^11. V. unverändert...

»14. V. Am Transplantat drei große, gezackte Stämme..

*17. V. Am Transplantat drei schöne, deutlich gezack te Stämme..*

^19. V. unverändert..*

»20. V. unverändert. .«

^26. V. Transplantat mit drei ungleich langen, stellenweise noch schwach gezackten Stämmen ..*

*3. VI. Am Transplantat schöne glatte, mittellange Stämme ..* „5. VI. Transplantat mit mittellangen freien Stämmen... 
77. VI. Am Transplantat schöne mittellange, freie Stämme ..

9. VI. Am Transplantat hinter Aufwölbung kmze Warzen . . *

*10. VI. 'Tlansplantat nur mehr glatte Aufwölbung . ."

Ich habe in diesem Beispiel alle auf das Transplantat beztiglichen ausdricklichen Bemerkungen des Protokolls wiedergegeben, um wenigstens in einem Falle die einzelnen Schritte der ersten and die scharf dagegen kontrastierende zweite Rückbildung aufzuzeigen. Auch hier handelte es sich um einen Fall, in dem der zweite, plötzliche Rückbildungsvorgang schon in ein Stadium fällt, in dem die anfängliche degenerative Riickbildung schon stehengeblieben ist und der neue ProzeB ganz selbständig einsetzt.

Ein weiteres Beispiel soll nun zeigen, daß dies keinesweg's immer so sein muB, sondern der zweite RückbildungsprozeB auch noch vor Eintritt des Dauerstadiums, also noch während des Andauerns der ersten Ruckbildung, einsetzen kann und daß er auch dann trotzdem als plötzliche, gewissermaßen vom Transplantat aus ganz unmotivierte Tempoänderung mit Sicherheit zu erkennen ist.

Es seien beispielweise einige Notizen wiedergegeben, die das Tier *III, 5 «; betreffen. Die Operation hatte am 26. III. 1913 stattgefunden. Am 12. IV. zeigte das Transplantat noch drei Stämme, von denen zwei groß und buschig gefiedert waren. Bei ungestörtem Verlauf der ersten Rückbildung wären, da sich das Transplantat mehr als zwei Wochen nach der Operation noch so auBerordentlich güustig verhielt, etwa im Verlauf von weiteren 4 Wochen die Fiedern langsam mehr oder weniger rïckgebildet worden, die Stämme hätten sich langsam etwas verkleinert, bis in der 6. bis 8 . Woche nach der Operation wohl das gewöhnliche Dauerstadium aufgetreten wäre, etwa mit dem Bilde: Am Transplantat drei dentliche, schwach gezackte Stämme verschiedener Länge usw. Statt dessen waren aber am 16. IV., also 4 Tage nach der zitiexten Beschreibung, am Transplantat hinter der Aufwölbung nur mehr zwei kleine Stummel zu sehen, die sich nun wieder etwas langsamer weiter zuriuckbildeten, warzenartig wurden und am 23. IV. ganz verschwunden waren. Hier wurde also die langsame erste Rtickbildung durch den zweiten Rückbildungsprozeß itberholt, die Trennung der beiden ist aber trotzdem, wie aus dem Gesagten deutlich hervorgeht, leicht möglich. Wichtig erscheint auch die Tatsache, daß hier deutlicher als sonst nach der den zweiten Prozeß darstellenden rapiden Rückbildung des größten Teiles der ubertragenen Kiemen die kleinen zackenartigen Reste erst wie- 
der langsamer vollständig rlickbildeten. Auf die Bedeutung dieser Erscheinung wird später noch eingegangen werden.

Diese Beispiele, die aus einer größeren Zahl gleicher Fälle willkürlich herausgegriffen wurden, mögen genügen, um die Trennbarkeit der beiden Rückbildungsprozesse und die gute Charakterisierbarkeit des zweiten Prozesses zu beweisen. Diese Verhältnisse, die in allen Fällen, bei denen sich das Transplantat in brauchbarer Gute erhielt, stets mèhr oder weniger deutliche Übereinstimmung zeigten, erlauben uns folgende Aussagen:

Im AnschluB an die Operation erfolgt in allen Fällen eine langsame, kontinuierliche, verschieden weitgehende Rückbildung an den ưbertragenen Kiemen, die in erster Linie die Kiemenfiedern, in geringerem Maße aber auch die Kiemenstämme betrifft. Ihr Verlanf erscheint vom Alter und Entwicklungszustand der zur Operation als Spender und als Empfänger verwendeten Tiere völlig unabhängig und nur durch die Operation bedingt. Die Ruickbildung durfte mit den bei Organverletzungen auftretenden Geweberiickbildungen in kausalem Zusammenhang stehen, von denen ror allem eine Verödung der Blutgefäße, wenigstens in den peripheren Teilen des Transplantates, histologisch nachweisbar ist. Ihr Stillstand, bzw. sogar eine gewisse Wiederaufdifferenzierung durfte mit der ebenfalls durch histologische Bilder wahrscheinlich gemachten Neuvaskularisierung des Transplantates vom Wirtstier her zusammenhängen. Es bandelt sich hier offenbar um eine durch die Operation hervorgerufene pathologische Erscheinung. Ich nenne den ProzeB dieser ersten Rückbildung darum den degenerativen Rickbildungsprozeß.

Außer dieser degenerativen Rtickbildung spielt sich am Transplantat ein zweiter Vorgang ab, der von dem Zeitpunkt der Operation und von dem durch die Operation bedingten Verhalten des Transplantates - eben der degenerativen Rückbildung - vollständig unabhängig ist. Wir müssen die Basis fur die Erklärung dieses zweiten Vorganges in der Eigenttimlichkeit des Transplantates, bzw. in der Eigentimlichkeit des Systems: Wirtsorganismus + Transplantat suchen.

Die nächste logische Frage lautet daher: Gibt es im normalen Verhalten der thertragenen Organe, $d . h$. in ihrem Verhalten unter den natürlichen Bedingungen einen Vorgang, der imstande ist, den am Transplantat erfolgenden zweiten Rückbildungsprozeß zu erklären, da dieser sich aus den direkten Folgen der Operation, also aus der Abänderung der natürlichen Bedingungen, nicht nur nicht erklären 
liißt, sondern sogar im Prinzip nichts mit ihnen zu tun zu haben scheint. Die Antwort auf diese Frage ist selbstverständlich. In der metamorphotischen Rückbildung der Kiemen unter normalen Umstïnden kennen wir einen Vorgang, der genau so verläuft wie der zweite : lickbildungsprozeB am Transplantat. Auch dort findet meist im selben Zeitraume von 1-3 Tagen eine rapide Rückbildung des größten Teiles der äußeren Kiemenanhänge statt, während eventuell nachher noch vorhandene zacken- oder warzenartige Reste erst wieder etwas langsamer vollständig verschwinden. Besonders auch dies letztere Verhalten zeigt auffallende Übereinstimmung mit dem Verhalten des Transplantates, wie zahlreiche Fälle zeigen. Auch unter den zitierten Versuchen findet sich diese Erscheinung, und zwar in den Fällen »III, $5 \ll ; » I I I, 9 * ; » I I, 4 *$ und »II, $2 \ll$.

Es erscheint mir darum vollkommen berechtigt, die bisher als *Zweiter RtickbildungsprozeB* bezeichnete rapide Rückbildung des größten Teiles der übertragenen Kiemen als einen metamorphotischen ProzeB, and zwar, da ja die Kiemen den Hauptteil des Transplantates bilden, auch direkt als Ausdruck der Metamorphose des Transplantates aufzufassen.

Nun wissen wir, daß sich unter naturlichen Verhältnissen die Kiemenrickbildung in zeitlichem Zusammenhang mit andern Veränderungen des Organismus abspielt. Der Kiemenrückbildungsprozeß verläuft nicht nur in den beiderseitigen Kiemen genau synchron, sondern es besteht auch, wie schon mehrfach hervorgehoben wurde, normalerweise Synchronie zwischen ihm and andern metamorphotischen Erscheinungen. Die Rlickbildung des größten Teiles der äußeren Kiemenanhänge erfolgt normalerweise gleichzeitig mit einer Häutung und mit einer starken, momentanen Beschleunigung in der Rückbildung des Ruderschwanzes kurz vor dem ans Landsteigen des Tieres.

Ist der zweite Ruckbildungsvorgang tatsächlich ein metamorphotischer, dann muissen sich diese Verhältnisse in seinem Verlauf änßern. Dies ist auch wirklich der Fall, ein weiterer Beweis für die Richtigkeit der Deutung. Ans der Art dieser Außerung aber können wir dann anderseits auf die Ursachen dieser Verhältnisse schließen. Denn von vornherein wäre, wie schon in der Literaturbesprechung anggefuhrt wurde, zweierlei denkbar. Die Synchronie unter normalen Verhältnissen könnte entweder einfach dadurch geregelt sein, daß von den in der gleichen Zeit unter gleichen Umständen verlaufenden Prozessen entsprechend diesen innerhalb des 
Individuums gleichen Bedingungen immer bestimmte Stadien in der Organentwicklung einander entsprechen und zeitlich miteinander verbunden erscheinen; und zwar wären dann nattllicherweise bei den gleichen Organen, die im Körper in Mehrzahl - etwa rechts und links - vorhanden sind, die einander »entsprechenden * Stadien auch einander gleich. Oder aber die Synchronie könnte durch einen spezifischen Einfluß geregelt sein, der dem Einzelorgan von der Gesamtheit der thbrigen Organe auf irgendeinem Wege ubermittelt wird. Es wurde in der Literaturbesprechung gezeigt, daß die Transplantationsmethode derartige Fragen in einer Reihe von Fällen entschieden hat, und zwar in dem Sinne, daß dort tatsächlich eine spezifische Beeinflussung des Einzelorganes durch die Gesamtheit der ubrigen Organe erfolgen müsse.

Auch in dem vorliegenden Falle müssen die Transplantationsergebnisse eine Entscheidung der Frage ermöglichen. Denn wenn die Synchronie nur durch das gleiche Alter und die gleiche Vergangenheit der Organe bedingt ist, dann miißte an den tubertragenen Kiemen eine metamorphotische Ritckbildung zu der Zeit stattfinden, in der sie auch normalerweise stattgefunden hätte, wenn die Kiemen am Spender belassen worden wären. Die beiderseitigen Kiemen eines Tieres müßten sich, auf zwei verschiedene andere Tiere versetzt, immer ungefähr gleichzeitig verwandeln, unabhängig vom Alter und Entwicklungszustand des Empfängers. Ist dagegen die metamorphotische Kiemenrïckbildung durch eine spezifische Einwirkung von der Gesamtheit der ubrigen Organe her geregelt, dann muß, wenn die Operation zu einem Zeitpunkt vorgenommen wird, in dem die Beeinflussung in den beiden Tieren noch nicht stattgefunden hat, am Transplantat eine Metamorphose erfolgen, die unabhängig vom Alter und Entwicklungszustand des Spenders genau synchron mit der Metamorphose des Wirtsorganismus erfolgt.

Etwas komplizierter liegen die Dinge, wenn die Operationen zu einer Zeit erfolgen, in der in einem der beteiligten Tiere die Beeinflussung schon stattgefunden hat. Doch haben die Ergebnisse UHLENHUTHs gezeigt, daß bei richtigen Arbeitsmethoden die dann erfolgenden heterochronen Metamorphosen gerade für die Deutung der Verhältnisse sehr wertvolle Dienste leisten können.

Bei den Kiementransplantationen nun erfolgte die metamorphotische Kiemenritckbildung am Transplantat immer genau synchron mit den metamorphotischen Erscheinungen am Wirtsorganismus. Als Beweis hierfür sollen wieder wörtliche 
Angaben der Protokolle dienen, wobei ich aber jetzt vor allem auf das Verhaltnis der Erscheinungen am Transplantat zu denen am Wirtsorganismus Gewicht lege.

Betrachten wir vor allem das Verhalten des Wirtsorganismus in den schon als Beweise der Trennbarkeit der beiden Rückbildungsprozesse herangezogenen Beispielen.

Bei dem Tier I, 12 war die metamorphotische Ruickbildung der Kiemenstämme am Transplantat am 30. III. konstatiert worden. Am selben Tage finden wir die das Wirtstier betreffende Angabe: " knapp vor der Verwandlung, eigene Kiemen ruckgebildet". Spätestens am 5. IV. hatte das Tier endgültig das Wasser verlassen.

Das Transplantat des Tieres II, 2 zeigt die metamorphotische Rtickbildung am 8. V. Das Wirtstier zeigte am 5. V. »beginnende. Rückbildung des Rudersaumes", am 6. V. waren »die eigenen Kiemen sehr kurz*, am 8. V. waren die eigenen Kiemen fast ganz rtickgebildet, der Rudersaum noch nicht völlig verschwunden, das Tier hatte aber schon das Wasser verlassen. Am 10. V., zur selben Zeit, in der auch die letzten zackenartigen Reste der transplantierten Kiemenstämme verschwunden waren, war auch die Verwandlung des Wirtstieres vollendet. (Soweit sie durch den hier immer allein betrachteten zeitlich zusammengehörigen Erscheinungskomplex gebildet wird: Häutung, Ruickbildung von Kiemen und Rudersaum, ans Landgehen.)

Das Transplantat von II, 4 metamorphosierte am 6. V.; am selben Tage war das Wirtstier ans Land gestiegen, die eigenen Kiemen völlig rilokgebildet, vom Rudersaum noch Reste vorhanden.

Das Transplantat von II, 6 hatte in der Zeit vom 26. IV. bis zum 1. V. seine Metamorphose vollendet. In derselben Zeit war das Tier ans Land gestiegen.

Das Transplantat von III, 9 zeigte eine metamorphotische Rückbildung am 9. VI, die Rickbildung der warzenartigen Reste am 10. VI. Das Wirtstier hatte rom 6. VI. zum 9. VI. den Rudersaum stark zuriickgebildet nud verlieb an diesem Tage schon zeitweise das Wasser; am 10. VI. war es endgilltig ans Land gestiegen. Allerdings hatten an diesem Tage die eigenen Kiemen noch kurze Stämme und Fiedern, und noch am 12. VI. waren von ihnen Reste in Form kurzer Zacken vorhanden. Dieser Fall erșcheint darum besonders interessant, weil er zeigt - und es ließen sich ihm noch andere Beispiele anfugen -, daß die Metamorphose des Transplantates, auch wenn sie infolge einer Beeinflussung durch den Wirtsorganis- 
mus mit dessen Gesamtmetamorphose auffallend deutlich synchron erfolgt, sie im einzelnen vielleicht in manchen Fällen nicht gerade genau synchron mit der Rickbildung der wirtseigenen Kiemen erfolgen muß, sondern, wie hier, eine noch strengere Synchronie mit andern Vorgängen des Komplexes (Rudersaumrückbildung, Verlassen des Wassers) vorliegen kann. Dies erscheint uns verständlich, wenn wir bedenken, daß in dem System Wirtsorganismus + Transplantat die eigenen und die transplantierten Kiemen sicher nicht in dem Sinne als identische Organe anfgefaßt werden durfen, wie etwa rechte und linke wirtseigene Kiemen. Es erfolgt eine Synchronie zwischen der Metamorphose des Transplantates und dem Erscheinungskomplex, der hier immer als einheitlich betrachtet wird, aber wie die Einzelvorgänge dieses Komplexes in einem gewissen, wenn auch sehr geringen Maße gegeneinander verschoben sein können, so auch die Metamorphose des Transplantates, die sich dadurch als den itbrigen Einzelkomplexen gleichgestellt, aber nicht dem einen derselben enger als dem andern verbunden erweist.

Das Einsetzen der metamorphotischen Kiemenrtickbildung, das bei alleiniger Wiedergabe der das Transplantat betreffenden Notizen völlig unmotiviert erscheint, zeigt also, wie aus diesen Daten hervorgeht, eine deutliche Übereinstimmung in seinem Ablauf mit dem Verlanf eines bestimmten Erscheinungskomplexes in der Metamorphose des Wirtsorganismus. Es bleibt nur noch zu beweisen, daß es sich erstens dabei nicht um irgendeine mechanische Beeinflussung des Transplantates durch die Vorgänge der Metamorphose handelt und zweitens, daß die Synchronie nicht etwa durch zufällige Übereinstimmung in der Entwicklungsgeschwindigkeit von Transplantat und Wirtsorganismus zustande kommen konute.

Beztiglich der Frage, ob es sich bei dem zweiten Ritckbildungsprozeß vielleicht doch um eine mehr äuBerliche Einwirkung handeln könnte, läßt sich folgendes sagen: Eine Beeinflussung durch die nach dem ans Landgehen geänderten Verhältnisse, also etwa ein Abtrocknen oder Abwetzen der frei abstehenden Kiemenstämme des Transplantates, ist ausgeschlossen, da die metamorphotische Ruckbildung stets vor dem ans Landgehen erfolgt. Eine Beeinflussung durch mehr äuBerliche Vorgänge vor dem ans Landgehen wäre meines Wissens nux noch eventuell dadurch möglich, daß das Tier bei der Häntung' die äußeren Teile des Transplantates mit abwirft. Aber auch das scheint mir ausg'eschlossen, und zwar aus folgenden Griuden: Erstens erfolgt die Haiutung durch einmaliges einheitliches Abwerfen einer 
ganzen Hautschicht, so daß man diese nachher noch zusammenhängend und oft noch in der Form des Tieres neben diesem im Wasser sieht. Das Verschwinden der Kiemenstämme am Transplantat dagegen erfolgt, wenn auch sehr rasch, doch nicht auf einmal, sondern schrittweise, wie aus zahlreichen Beobachtungen hervorgeht. Zweiteus scheint $\mathrm{z}$ wischen Metamorphose des Transplantates und Häutung des ganzen Tieres kein innigerer zeitlicher Zusammenhang zu bestehen, als unter den ibrigen metamorphotischen Erscheinungen desselben Komplexes, und dieser ist, wie schon betont, verschiebbar, wenn auch nur innerhalb enger Grenzen. Endlich aber überzeugt meiner Ansicht nach auch das anatomische Verhalten des Transplantates von der Unwahrscheinlichkeit der Annahme, daß sich bei der Häutung die ganzen freien Stämme des Transplantates von dessen übrigen Teilen etwa aus irgendwelchen mechanischen Grinden loslösen sollten. Denn das die Stämme füllende Bindegewebe geht ohne Trennung direkt in das Bindegewebe des Höckers und dieses direkt in das des Wirtstieres ûber. Zwischen den inneren Teilen der freien Stämme und den am Wirtstier bei der Metamorphose bleibenden Teilen besteht daher durch die Verwachsung ein in rein mechanischer Beziehnng wohl viel festerer Zusammenhang, als $\mathrm{zwischen}$ den bei der metamorphotischen Ruckbildung verschwindenden Kiemenstämmen des Transplantates und der bei der Häutung: abgeworfenen Schicht des Wirtstieres.

Aus allen diesen Grüden halte ich es für ausgeschlossen, daB an dem metamorphotischen Kiemenrïckbildungsprozeß am Transplantat etwa doch irgendein äußerlich wirkender mechanischer Faktor Schuld tragen könnte. Vielmehr halte ich es fur bewiesen, daß es sich tatsächlich um einen echten metamorphotischen Entwicklungsvorgang des Transplantates handeln muß.

Endlich ist noch der eindeutige Beweis daftur zu erbringen, daB die metamorphotische Kiemenritckbildung am Transplantat wirklich nur von der Metamorphose des Wirtsorganismus abbängig;, vom Alter und Entwicklungszustand des Spenders dagegen vollständig unabhängig erfolgt, daß es sich also nicht etwa um zufällige Übereinstimmung in der Entwicklungsgeschwindigkeit von Transplantat und Wirtstier handeln könne.

Wie schon bei Besprechung der Versuche von BoRN bemerkt wurde, kann als Wahrscheinlichkeitsbeweis hierfur schon die synchrone Verwandlung bei Verwendung gleich alter Tiere dienen, da sich anch gleich alte Tiere selbst eines Wurfes und auch bei Hal- 
tung unter möglichst gleichen Bedingungen stets zu ganz verschiedenen Zeiten verwandeln. Der sichere Beweis dafür aber liegt in den in der III. Serie erhaltenen Resultaten der Versuche, bei denen als Spender und als Empfänger verschieden alte Tiere verwendet wurden.

Es sei hier beispielweise der schon erwähnte Fall III, 5 genannt. Die Verwandlung des Transplantates erfolgte genau synchron mit der des Wirtstieres in der Zeit: 16. IV.-20. IV. Das Wirtstier war zu dieser Zeit 6 Monate alt. Das Transplantat entstammte einem Spender, der am 6. III. dem Uterus entnommen war, verwandelte sich also nach einer Zeit von $6 \mathrm{~W}$ Wohen! Selbstverständlich war za dieser Zeit noch keines von den Geschwistertieren des Spenders in Metamorphose. Das erste verwandelte sich am 22. V., also 21/2 Monate nach der Uterusentnahme. Dies war schon als Ausnahmefall zu betrachten. Denn die weitaus meisten Tiere dieses. Wurfes verwandeIten sich erst mehrere Wochen später (siehe auch Tabelle I). Die Verwandlung des Transplantates erfolgte also um 5 Wochen fruher, als die früheste unter den Geschwistertieren des Spenders. Hier erfolgte also sicher eine Beschleunigung der Verwandlung des Transplantates, die unter dem Einfluß des Wirtstieres zu genaner Synchronie führte.

Ähnlich waren auch die Fälle III, 12; III, 18; III, 22. In allen dreien erfolgte die Verwandlung des Transplantates, das Tieren desselben Wurfes entnommen worden war wie bei III, 5 , fruiher als bei den weitergeztichteten Tieren desselben Wurfes, nämlich am 25. bis 28. IV.; am 9.-10. V. und am 10.-14. V. Auch hier lag also sicher eine beschleunigte Metamorphose des Transplantates vor, herbeigefuhrt durch das größere Alter der Empfänger, die auch demselben Wurf entstammten wie der Empfänger III, 5.

Ebenso lag'en die Verhältnisse auch bei der Verwendung von Larren, die am 14. IV. 1913 geworfen worden waren, als Spender. Im Falle III, 28 waren die Kiemen eines solchen Tieres auf ein am 21. X. 1912 geworfenes verpflanzt worden. Die Verwandlung des Transplantates erfolgte in der Zeit vom 17.-22. V. genau synchron mit der Verwandlung des Wirtstieres. Das erste Geschwistertier des Spenders verwandelte sich am 10 . VII., also fast 3 Monate nach dem Wurf, mehr als $1 \frac{1}{2}$ Monate später als das erwähnte Transplantat, die andern Tiere des Wurfes waren auch zu dieser Zeit noch rein larval. Auch bei Verwendung dieses Wurfes als Spender und der am 6. III. dem Uterus entnommenen Larven als Empfänger trat noch 
eine sichere, nachweisbare Beschleunigung ein. Das Transplantat von III, 26 verwandelte sich synchron am 22. V., also mehr als 11/2 Monate vor dem ersten Geschwistertier des Spenders. Ahnlich noch das Transplantat von III, 27 am 17. VI.

So wie in allen diesen Beispielen eine sichere Beschleunigung: vorlag, erfolgte in den umgekehrten Fällen eine sicher nachweisbare Verzögerung, die allerdings nicht so auffallend ist, weil dem längeren Verweilen im Larvenzustand viel weniger enge Grenzen gesetzt sind, als der frühzeitigen Metamorphose. Als Beispiel mög'e folgender Fall dienen:

Die Verwandlung der am 21. X. 1912 entnommenen Larven erfolgte in der Zeit zwischen 10. IV. und 20. VI. 1913, die der meisten Larven etwa Ende Mai. Wurden diese Larven als Spender, die am 6. III. 1913 dem Uterus entnommenen Larven als Empfänger verwendet, dann war die Verzögerung der Verwandlung manchmal deutlich konstatierbar. So verwandelte sich das Transplantat von III, 13 zwischen 26. VI. und 29. VI., also später als die spätesten Geschwister des Spenders.

Besonders itberzeugend erscheint mir die Gegentiberstellung des Verhaltens yon Transplantaten, bei denen die beiderseitigen Kiemen eines Spenders je auf zwei verschieden alte Empfänger verpflanzt wurden. Bei diesen Fällen sieht man, wie sich rechte und linke Kiemen eines Individuums zu verschiedenen Zeiten verwandeln, je nach dem Alter des Empfängers. Diese Verhältnisse gehen am klarsten aus der Tabelle II hervor, in der solche Fälle zusammengestellt sind.

Nur ein derartiges Beispiel soll hier noch näher besprochen werden, weil es gleichzeitig bisher der einzige Fall ist, der fitr die hauptsächlich in der IV. Versuchsserie anfgeworfene Frage einigermaßen verwendbare Resultate lieferte, für die Frage, wie weit das Halten im feuchten Raum statt im Wasser das Verhalten transplantierter und wirtseigener Kiemen beeinflusse. In den andern derartigen Versuchen, besonders also in denen der IV. Serie, gingen die Larven zu schnell zugrunde, als daB ein deutliches Resultat erkennbar gewesen wäre.

Von einem am 6. III. 1913 dem Uterus entnommenen Tiere wurden am 9. IV. die Kiemen der einen Seite auf ein gleich altes, die der andern Seite auf ein am 21. X. 1912 dem Uterus entnommenes Tier ibertragen (III, 11 und III, 12). Die beiden Transplantate verhielten sich gleich etwas verschieden, indem auf dem älteren Emp- 
fänger* die degrenerative Ruokbildung etwas schneller fortschritt als auf dem jungeren. Ob dies Zufall war oder auf Grinde von allgemeiner Bedeutung zurickgeht, kann ich nicht mit Sicherheit entscheiden. Man könnte daran denken, daß die der Metamorphose des Empfängers vorhergehenden Stadien auch die degenerative Ritckbildung des Transplantates beginstigen, oder daian, daß die Erscheinung in Parallele zu setzen sei, mit der normalerweise vor der plötzlichen Rückbildung des größten Teiles der Kiemen vor sich gehenden langsamen, geringfügigen Verkleinerung der Kiemen. Nach meinen ubrigen Beobachtungen scheint es mir aber noch wahrscheinlicher; daB daran einfach der Umstand Schuld trägt, daB gleiches Alter, wie jede andere Gleichheit von Eropfänger und Spender, giunstigere, ungleiches Alter, wie jede andere Ungleichheit, unginstigere Bedingungen für die Transplantation darstellen. Immerhin reichte das Verhalten des Transplantates auf dem älteren Tiere (III, 12) vollkommen aus, um die bald darauf stattfindende synchrone Metamorphose zu demonstrieren: Denn am 22. IV. waren an diesem Transplantat noch «2 Stämme mit unregelmäBigen Konturen und Zacken am 23. IV. »2 unförmige, gezackte Stämmchen «, am 24. IV. an diesen "noch deutliche mittellange Fiedern* zu sehen. Am Wirtsorganismus waren an den schon in langsamer Ruckbildung begriffenen Kiemen am 23. IV. als Fiederreste nur noch kurze Zacken vorhanden, der Rudersaum war fast ganz rỉckgebildet. Am 25. IV. hatte das Wirtstier sich gehäutet, von seinen Kiemen waren nur mehr die Stämme erhalten. Das Transplantat zeigte noch s deutlich gezackte Stummeln*; am 26. IV. „nur noch ganz kleine ausgezackte Stummeln*: Am: 28. IV. stieg das Tier ans Land, das Transplantat. zeigte von den Kiemenanhängen keine Reste mehr und war, wie gewöhnlich, nur noch als glatte Aufwölbung zu erkennen.

Vergleichen wir hiermit nun das Verhalten des juingeren Tieres (III, 11). Sein Transplantat zeigte am 22. IV. noch schöne lange Fiedern. An diesem Tage wurde es nun aus dem Wasser genómmen. und weiter in einer Schale mit feuchtem Filtrierpapier gehalten. Trotzdem spielten sich sowohl an seinen eigenen als auch an den transplantierten Kiemen nur ganz langsame und allmähliche Veränderungen ab. Es zeigte am 28. IV. noch rein larvale Charaktere, dasTransplantat hatte an diesem Tage, an dem das Transplantat von III, 12 in synchroner Metamorphose die letzten Kiemenreste zurtickgebildet hatte, noch drei schöne, ausgezackte Stämme. Am 1. V. waren am Transplantat noch drei schöne glatte (also ohne Fiederreste) 
Stämme erhalten, am 10. V. noch $\star$ drei deutliche, glatte mittellange. Stämme*, Die wirtseigenen Kiemen hatten wohl noch lange Fiedern, zeigten aber doch auch schon Rtickbildungserscheinungen. Am 17. V. waren von ihnen nur noch mittellange Stämme mit kurzen Fiedern und großen Zacken zu sehen. Das Transplantat hatte an diesem Tage drei deutliche, aber sehr kurze Stämme«, die am 22. V. kaum mehr zu sehen, am 26. V. scheinbar ganz verschwunden, am 5. VI. bei genauerer Untersuchung doch noch als kleine Reste nachweisbar waren. Die wirtseigenen Kiemen hatten am 22. V. "mittellange Stämme mit verscbieden langen Zacken, am 26. V. waren auch hier die Zacken fast verschwunden, doch waren am 5. VI. bei genauerer Untersuchung noch schwache Fiederreste nachweisbar. Am 7. VI. ging das Tier ein.

Es zeigt sich also, daß das Halten in der feuchten Kammer und die dadurch hervorgerufene Schädigang des ganzen Organismus an den wirtseigenen und an den transplantierten Kiemen ziemlich ähnliche Wirkungen hervorruft. Am Transplantat erhält man den Eindruck, daß die degenerative Ruckbildung in etwas gesteigertem Maße fortschreitet und nicht zu einem Stillstand gelangt, dabei aber in keiner Weise der metamorphotischen Ruckbildung ähnlicher wird. An den wirtseigenen Kiemen spielt sich ein Vorgang ab, welcher der degenerativen Ruckbildung am Transplantat in höchstem Maße gleicht, vor allem die Fiedern zur Ruckbildung bringt, aber auch die Stämme stark angreift. Wir können daraus entnehmen, daB die degenerative Rückbildang eine Erscheinung ist, mit der die Kiemen immer in gleicher Weise auf die verschiedenartigsten Schädigungen reagieren. Vielleicht könnte auch die anfängliche langsame Ruckbildung normaler Kiemen vor der Metamorphose hierher gerechnet werden. Auf prinzipiell davon verschiedene Grtade sind offenbar die eigentlich metamorphotischen Kiemenruckbildungen zur tuckzufuhren, deren Verlauf auch einen ganz andern Charakter zeigt.

Eine den UnLenHUTHschen und eventuell auch den WrigLschen Resultaten entsprechende heterochrone Kiemenrückbildung bei Verwendung besonders alter Larven konnte bisher in keinem einzigen Falle einwandfrei nachgewiesen werden. Das kann entweder ein Zufall sein, indem nicht die richtigen Stadien zur Verwendung kamen, es könnte aber auch seinen Grund darin haben, daß die EinfluBnahme des Gesamtorganismus auf die einzelnen metamorphosierenden Organe zu verschiedenen Zeiten, vielleicht sogar auf verschiedene 
Abhängigkeit der metamorphotischen Kiemenrïckbildung usw.

Weise erfolgt, bei den Kiemen beispielsweise erst sehr spät, erst nach der Beeinflussung der Augen und der Haut. Es spricht dafür jedenfalls die meiner Ansicht nach feststellbare relativ große Unabhängigkeit der Augen- und der Hautmetamorphose von dem Komplex: Häntung, Rudersaumrtickbildung, Verlassen des Wassers, Kiemenriickbildung, bei relativ groBer Abhängigkeit der in diesem Komplex zusammengefaßten Erscheinungen untereinander. Ea ist zu hoffen, daß über diese und ähnliche interessante Fragen weitere Versuche, besonders kombinierte Transplantationen, Anfklärung bringen werden.

\section{Zusammenfassung der Ergebnisse.}

An Larven von Salcmandra maculosa wurden homöoplastische Kiementransplantationen vorgenommen, die folgende Resultate ergaben:

1) Am Transplantat spielt sich zuerst eine gleich nach der Operation einsetzende, langsame, kontinuierliche degenerative Ruckbildung ab, die aber nach einiger Zeit zum Stillstand kommt.

2) Von dieser degenerativen Ruckbildung ist ein anderer Rückbildungsprozeß scharf zu trennen, der zu einer vollständigen Rlickbidung aller noch vorhandenen freien Kiemenanhänge des Transplantates innerhalb eines sehr kurzen Zeitraumes fuhrt. Er ist im Gegensatz zu der degenerativen Riickbildang von. dem Zeitpunkt der Operation und von dem Verhalten des Transplantates nach derselben völlig unabhängig: und kann in jedem Stadium, der degenerativen Rlickbildung sowie auch des auf sie folgenden Dauerstadinms einsetzen. $\mathrm{Er}$ ist als metamorphotische Kiemenrtickbildung, zugleich auch als Ausdruck der Metamorphose des Transplantates uberhaupt, aufzufassen.

3) Diese metamorphotische Kiemenrtickbildung erfolgt stets genau synchron mit der Metamorphose des: Wirtstieres und beweist so, daß offenbar auch unter normalen Umständen die Kiemenrtickbildung in ihrem zeitlichen Verlauf durch den Gesamtorganismus bestimmt wird.

4) Heterochrone Metamorphose der Kiemen bei Verwendung besonders weit entwickelter Larvenstadien konnte bisher nicht beobachtet werden. 


\section{Tabelle I.}

Die synchronen Metamorphosen der Transplantate der III. Serie.

Die Tabelle zeigt die Verschiebung des Zeitpunktes der Metamorphose je nach dem Alter des Empfüngers.

\begin{tabular}{|c|c|c|c|c|}
\hline \multirow{2}{*}{$\begin{array}{c}\text { Geburt des } \\
\text { Sponders }\end{array}$} & : & \multicolumn{3}{|c|}{ Geburt des Enipfängers } \\
\hline & & 21. X. 1912 & 6. III. 1013 & 14. IV. 1913 \\
\hline 21. X. 1912 & $\begin{array}{l}\text { synchrone Metan } \\
\text { 11. IV. }-20 . \mathrm{IV} . \\
\text { 21. IV. - 30.IV. } \\
\text { 1. V. - 10. V. } \\
\text { 11. V. - 20. V. } \\
\text { 21. V. - 30. V. } \\
\text { 1. VI. - 10. VI. } \\
\text { 11. VI. - 20. VI. } \\
\text { 21. VI. - 30. VI. } \\
\text { 1. VII. - 10. VII. } \\
\text { ain 11. VII. nocl larval }\end{array}$ & $\begin{array}{l}\text { irphose de } \\
\text { isplantate } \\
\text { - }\end{array}$ & $\begin{array}{c}\text { mpfänger } \\
\mathbf{3} \\
\\
-\end{array}$ & $\cdot$ \\
\hline 6. III. 1913 & $\begin{array}{c}\text { 11. IV. }-20 . \mathrm{IV} . \\
\text { 21. IV. }-30 . \mathrm{IV} . \\
\text { 1. V. }-10 . \mathrm{V} . \\
\text { 11. V. }-20 . \mathrm{V} . \\
\text { 21. V. }-30 . \mathrm{V} . \\
\text { 1. VI. }-10 . \mathrm{VI} \\
\text { 11. VI. }-20 . \mathrm{VI} . \\
\text { 21. VI. - 30. VI. } \\
\text { 1. VII. - 10. VII. } \\
\text { am 11. VII. noch larval }\end{array}$ & 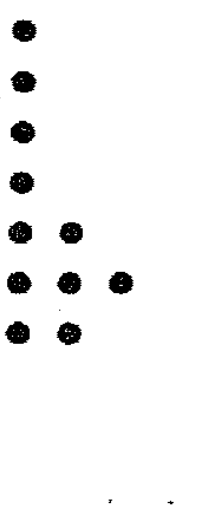 & $\bullet \bullet$ & 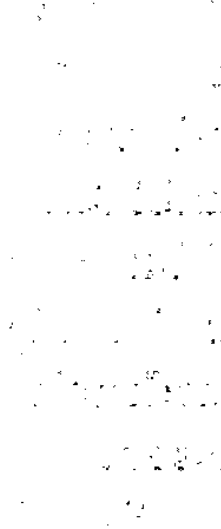 \\
\hline 14. IV. 1913 & $\begin{array}{c}\text { 11. IV. }-20 . \mathrm{IV} . \\
\text { 21. IV. }-30 . \mathrm{IV} . \\
\text { 1. V. }-10 . \mathrm{V} . \\
\text { 11.V. }-20 . \mathrm{V} . \\
\text { 21. V. }-30 . \mathrm{V} . \\
\text { 1. VI. }-10 . \mathrm{VI} \\
\text { 11. VI. - 20. VI. } \\
\text { 21. VI. - 30. VI. } \\
\text { 1. VII. - 10. VII. } \\
\text { am 11. VII. noch larval }\end{array}$ & $\cdots$ & 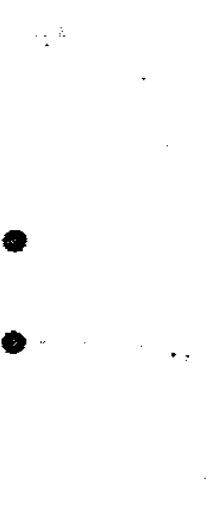 & 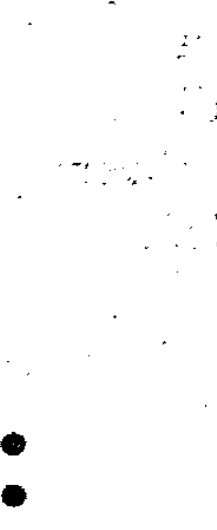 \\
\hline
\end{tabular}




\section{Tabelle II.}

Synchrone Verwandlung ron Transplantaten, bei denen je die beiderseitigen Kiemen eines Spenders auf zwei verschieden alte Empfänger tibertragen wurden. 1912/13.

\begin{tabular}{|c|c|c|c|c|}
\hline & Empfänger $\mathrm{T}$ & Spender & Empfünger II & \\
\hline Geburt & 6. III. & 6. III. & 21. $\mathrm{X}$. & Geburt \\
\hline Netamorphose & bis 7. VI. InrvaI & $\begin{array}{l}\text { der Geschwister: } \\
\text { nach dem } 21 . \mathrm{V} \text {. }\end{array}$ & 28. IV.! & Metamorphose \\
\hline Geburt & 6. III. & $21 . \mathrm{X}$ & 21. $\mathrm{x}$. & Geburt \\
\hline Netamorphose & 29. VI.! & $\begin{array}{l}\text { der Geschwister: } \\
\text { 10. IV. - } 20 . \mathrm{VI} \text {. }\end{array}$ & 22. IV. & Metamorphose \\
\hline Geburt. & 6. III. & 21. X. & 21. $\mathrm{x}$. & Geburt \\
\hline Metamorphose & 5. VI. & $\begin{array}{l}\text { der Geschwister: } \\
\text { 10.IV. - } 20 . \mathrm{VI} \text {. }\end{array}$ & 10. V. & Metamorphose \\
\hline Geburt & 6.III. & 6.III. & 21. X. & Geburt \\
\hline Metamorphose & bis 9. VII. larval & $\begin{array}{l}\text { der Geschwister: } \\
\text { nach dem } 21 . \mathrm{V} .\end{array}$ & 11. V..! & Metamorphose \\
\hline Geburt & 6. III. & 6. III. & 21. $X$ & Geburt \\
\hline Metamorphose & bis 9. VII. larval & $\begin{array}{l}\text { der Geschwister: } \\
\text { nach dem } 21 . \mathrm{V} \text {. }\end{array}$ & ธ. VI. & Metamorphose \\
\hline Geburt & 6. III. & 6. III. & 21. $X$. & Gebart \\
\hline Metamorphose & 21. VI. & $\begin{array}{l}\text { der Geschwister: } \\
\text { nach dem } 21 . \mathrm{V} \text {. }\end{array}$ & 14. V.! & Metamorphose \\
\hline $\begin{array}{c}\text { Geburt } \\
\text { Metamorphose }\end{array}$ & $\begin{array}{l}\text { 6. III. } \\
\text { 17. VI.! }\end{array}$ & $\begin{array}{l}\text { 14.IV. } \\
\text { der Geschwister: } \\
\text { nach dem 10. VII. }\end{array}$ & $\begin{array}{l}\text { 21. } \mathrm{X} \\
25 . \mathrm{V},\end{array}$ & $\begin{array}{c}\text { Geburt } \\
\text { Metamorphose }\end{array}$ \\
\hline
\end{tabular}

\section{Literaturverzeichnis.}

BoAs, J. E. V., Utber den Conus arteriosus und die Arterienbogen der Amphibien. Morph. Jahrb. Bd. 7. 1882.

- Beitrige zur Angiologie der Amphibien. Morph. Jahrb. Bd. 8. 1883.

Bors, G., Ửber Verwachsungsversuche mit Amphibienlarven. Arch. f. Entw.Meoh. Bd. 4. 1897.

BRESCA, G., Experimentelle Untersuchungen tiber die sekundüren Sexualcharaktere der Tritonen. Arch. f. Entw.-Mech. Bd. 29. 1910.

Cirrid, C. M., A study of senescence and rejuvenescence, based on experiments with Planarians. Arch. f. Entw.-Mech. Bd. 31. 1911.

Chemens, P., Die äuBeren Kiemen der Wirbeltiere. Anat. Hefte. I. Abt. Bd. 5. 1895.

Cramptox, H. E., jun., Coalescence Experiments upon Lepidoptera. Woods Hall Biolog. Lectures for 1896/97. Boston 1897. 
Crampton, H. E., jun., An Important Instance of Insect Coalescence. Annals New York Acad. Seience. Vol. 11. p. 219. Lancaster P. A. 1898.

- An Experimental Study upon Lepidoptera. Arch. f. Entw.-Mech. Bd.9. 1899.

Erman, G., Experimentelle Untersuchungen über die Entwicklung der Kiemenregion usw. Morph. Jahrb. Bd. 47. 1913 a.

Über die Entstehung von Kiemenfäden und Kiemenspalten aus transplantiertem ortsfremden Ertoderm bei Bombinator. Morph. Jahrb. Bd. 47. 1913 b.

Grafe, V., und LrNsBaUER, $\mathbb{K}$., Über die wechselseitige Beeinflussung von Nicotiana. Ber. d. Deutsch. bot. Ges. Bd. 24. 1906.

GUIGNARD, Recherches physiologiques sur la greffe des plantes a acide cyanhydrique. Annales des Sciences Naturelle Botanique. 9e Série. Tom.6. 1907. Zitiert nach Meyer u. Sohmiot, 1910.

GUrwitsch, A., Untersuchungen über den zeitlichen Faktor der Zellteilungen. 2. Mitteilnng: Über das Wesen und das Vorkommen der Determination der Zellteilung. Arch. f. Entw.-Mech. Bd. 32.1911.

Guthrie, C. C., Further Results of Transplantation of Ovaries in Chickens. Journ. of Exp. Zool. Vol. V. 1907.

HARRISON, R. G., Experimentelle Untersuchangen über die Entwicklung der Sinnesorgane und der Seitenlinie der Amphibien. Arch. f. mikr. Anat. Bd. 43. 1904 .

HARMS, W., Beeinflussung der Daumenballen des Kastraten dureh Transplantation anf normale Rana fusca. Zool. Anz. Bd. 39. 1912.

HeRBST, C., Vererbungsstudien. VII. Die cytologischen Grundlagen der Verschiebung der Vererbangsrichtung nach der mütterlichen Seite. 2. Mitteil. Arch. f. Entw.-Mech. Bd. 34. 1912.

KAMMERER, P., Vererbung erzwungener Farbanpassungen. IV. Arch. f. Entw.Mech. Bd. 36. 1913.

Kopé, STr, Untersuchungen über Kastration und Transplantation bei Schmetterlingen. Arch. f. Entw.-Mech. Bd. 33. 1911.

KonNFeLD, W., Uber Kiementransplantationen an Salamanderlarven. Biolog. Zentralbl. Bd. 33. 1913.

Korsonelt, E., Regeneration und Transplantation. Jena 1907.

VAN LeERSUm, Over den invloed de Cinchona ote. Natunrkundig Tijdschr. van Ned. Ind. Deel 59. 1900. Zitiert nach Meyer u. SoHmidt, 1910.

LewIS, G. H., Experimental studies on the development of the eye in Amphibia. Americ. Jouru. of Anat. Yol. 3. 1904. Zitiert nach ScHÖNe, 1912.

LINDEMUTH, H., Über vegetative Bastardierung durch Impfung. Landwirtschaftl. Jahrbiicher. Bd. 7. 1877. Zitiert nach Konscrrox, 1907.

LOEB, J., Hat das Zentralnervensystem einen EinfuB auf die Vorgänge der Larvenmetamorphose? Arch. f. Entw.-Mech. Bd. 4. 1896.

LOEB, L., Beiträge zur Analyse des Gewebewachstums. IV. Über den Einfluß von Kombinationsreizen auf das Wachstum des transplantierten Uterus des Meerschweinchens. Arch. f. Entw.-Mech. Bd. 31. 1911.

Marohand, F., Der ProzeB der Wundheilung. Deutsche Chirargie. 1901. Zitiert nach SCHÖNE, 1912.

Maurer, F, Schilddrise, Thymus und Kiemenreste der Amphibien. Morphol. Jahrb. Bd. 13. 1888 a.

- Die Kiemen und ihre Gefäße bei anuren und urodelen Amphibien usw. Morph. Jahrb. Bd. 14. 1888 b. 
Meisenheimer, Ergebnisse einiger Versuchsreihen tiber Exstirpation tund Transplantation der Geschlechtsdrusen bei Schmetterlingen. Zool. Anz. Bd. 32. 1907.

- Über den Zusammenhang von Geschlechtsdrisen und sekundären Geschlechtsmerkmalen bei Arthropoden. Verh, d. Deutsch. Zool. Ges. 1908.

Meyer, A., und Schmot, E., Über die gegenseitige Beeinflussung der Symbionten heteroplastischer Transplantationen, mit besonderer Berücksichtigung der Wanderung der Alkaloide durch die Pfropfstellen. Flora. Bd, 100. 1910. Merns, R., Transplantation embryonaler und jugendlicher Keimdrüsen auf erwachsene Individuen bei Anuren, nebst einem Beitrag über Transplantationen geschlechtsreifer Froschhoden. Arch. f. mikr. Anat. Bd. 79. 1912.

Moens, J. C. B., De Kinacultur in Azie. Batavia, Ernst u. Cie. 1882. Zitiert nach Meyer und Schmid, 1910.

OPPEL, A., Lehrbuch der vergl. mikrosk. Anatomie d. Wirbeltiere. VI. Atmungsapparat. Jena 1905.

Przibram, H., Experimentalzoologie. II. Regeneration. Leipzig und Wien 1909.

— III. Phylogenese. 1910. S. 18.

- IV. Vitalität. 1913.

Rehi, E., und Warabayaschi, Die homöoplastische Transplantation im Tierexperiment. Arch. f. klin. Chirurgie. Bd. 97. 1912.

RiBbert, H., Über Transplantation von Ovarium, Hoden und Mamma. Arch. $f$. Entw.-Mech. Bd. 6. 1897.

SchöNe, G., Die heteroplastische und homboplastische Traneplantation im Tierexperiment. Berlin 1912.

Sorokina, M., Über Synchronismus der Zellteilungen. Arch. f. Entw.-Mech. Bd. 35. 1912.

Steinadr, E., Willkürliche Umwandlung von S̈̈ugetier-Männchen in Tiere mit ausgeprägt weiblichen Geschlechtscharakteren usw. PrLüGens Arch. Bd.144. 1912.

- Feminierung von Männchen und Maskulierung von Weibchen. Zentralblatt f. Physiologie. Bd. 27. 1913.

StrasburGer, E., Über Verwachsung und deren Folgen. Ber d. Deutsch. bot. Ges. 1885.

— Z $\mathrm{dem}$ Atropinnachweis in iden Kortoffelknollen. Ber. d. Deutsch. bot. Ges. 1906.

Uhlenhuth, E., Die Transplantation des Amphibienauges. Arch. f. Entw.-Mech. Bd, 33. 1912.

- Die synchrone Metamorphose transplantierter Salamanderaugen. Arch. f. Entw.-Mech. Bd. 36. 1913 a.

- Der Einfluß des Wirtes auf das transplantierte Amphibienauge. Arch. f. vgl. Ophthalmologie. Bd. III. $1913 \mathrm{~b}$.

Vöohring, H., Über Transplantation am Pflanzenkßrper. Tuibingen 1892.

WEIGI, R, UUber homöoplastische und heteroplastisehe Hauttransplantation bei Amphibien mit besonderer Berücksichtignng der Metamorphose. Arch. $f$. Entw.-Mech. Bd. 36. 1913.

Winkler, F., Stadien über Pigmentbildung. II. Transplantationsversuche an pigmentierter Haut. Arch. f. Entw.-Mech. Bd. 29. 1910.

Wintrebert, P., Sur la métamorphose de Salamandra maculosa Laur. dans les régiong privées du système nerveuse médullaire. Compt. rend. Soc. biol. Paris. Ir. Année. 1905. 
Wintrentert, $P$, - Sur la régression de la queue en l'absence des centres médullaires chez Rana viridis. Compt. rend. Soc. biol. Paris. 1905. II.

Sur l'accomplissement régulier des fonctions de nutrition, des processus d'ontogénẻse, de régénération et de métamorphose chez des larves d'Alytes, en l'absence d'une grande étendue de la moëlle. Ebenda. 1906. I.

- La métamorphose de Salamandra maculosa Laur. en dehors do la moëllo et des ganglions spinaux. Étude histologique. Ebenda. 1906. I.

Sur le déterminisme de la métamorphose chez les amphibiens. XX. La regression de la quene en dehors du système nerveux lateral, chez Alytes obstetricans. Ebenda. 1911.

\section{Erklärung der Abbildungen.}
St Stamm,
$F$ Fiedern,
G Blutgefäß,
$M$ Muskeln,
$B$ Bindegewebe,

Abkürzungen.

$$
\begin{array}{ll}
\text { G.D } & \text { Giftdrüsen, } \\
T & \text { Thymus, } \\
I & \text { Kiemenbogenknorpel, } \\
M & \text { Mitosen. }
\end{array}
$$

Die Figuren 1-8 illustrieren das morphologische Verhalten des Transplantates.

Die Figuren wurden mit Benutzung des ABBsschen Zeichenapparates und der Präparierlupe bei etwa achtfacher Vergrößerung gezeichnet.

\section{Tafel IX und $\mathbf{X}$.}

Fig. 1. Spender nach Entnahme des Transplantates. (Vorderkörper in Seitenansicht.)

Fig. 2. Empfänger mit der zur Anfnahme des Transplantates bestimmten Wunde (Halsregion in Dorsalansicht).

Fig. 3. Ein eben abgetragenes Transplantat.

Fig. 4. Tier mit 2 Tage altem Transplantat. Die transplantierten Kiemen zeigen noch vollkommen normales Aussehen. (Vorderkörper in Rïckenansicht.)

Fig. 5. Versuch III, 30. Operation 24. IV.; zwischen gleich alten (6 Wochen alten) Larven, am 9. VII. zeigt das Transplantat nach abgeschlossener degenerativer Rückbildung im Dauerstadium drei schöne Stämme mit Fiederresten. (Vorderkörper in Riickenansicht.)

Fig. 6. Versuch II, 14. Transplantat im Dauerstadium, $41 / 2$ Monate nach der Transplantation. Die wirtseigenen Kiemen sind gestutzt. (Halsregion, linke Hälfte in Ruickenansicht:)

Fig. 7. Versuch I, 1. Über 9 Monate altes Transplantat im Dauerstadium. (Vorderkörper in Riickenansicht.)

Fig. 8. Verwandeltes Tier. Kiemen des Transplantates synchron rückgebildet. (Halsregion in Seitenansicht.)

Die Figuren 9-21 zeigen nach Schnitten das histologische Verhalten normaler und transplantierter Kiemen. 


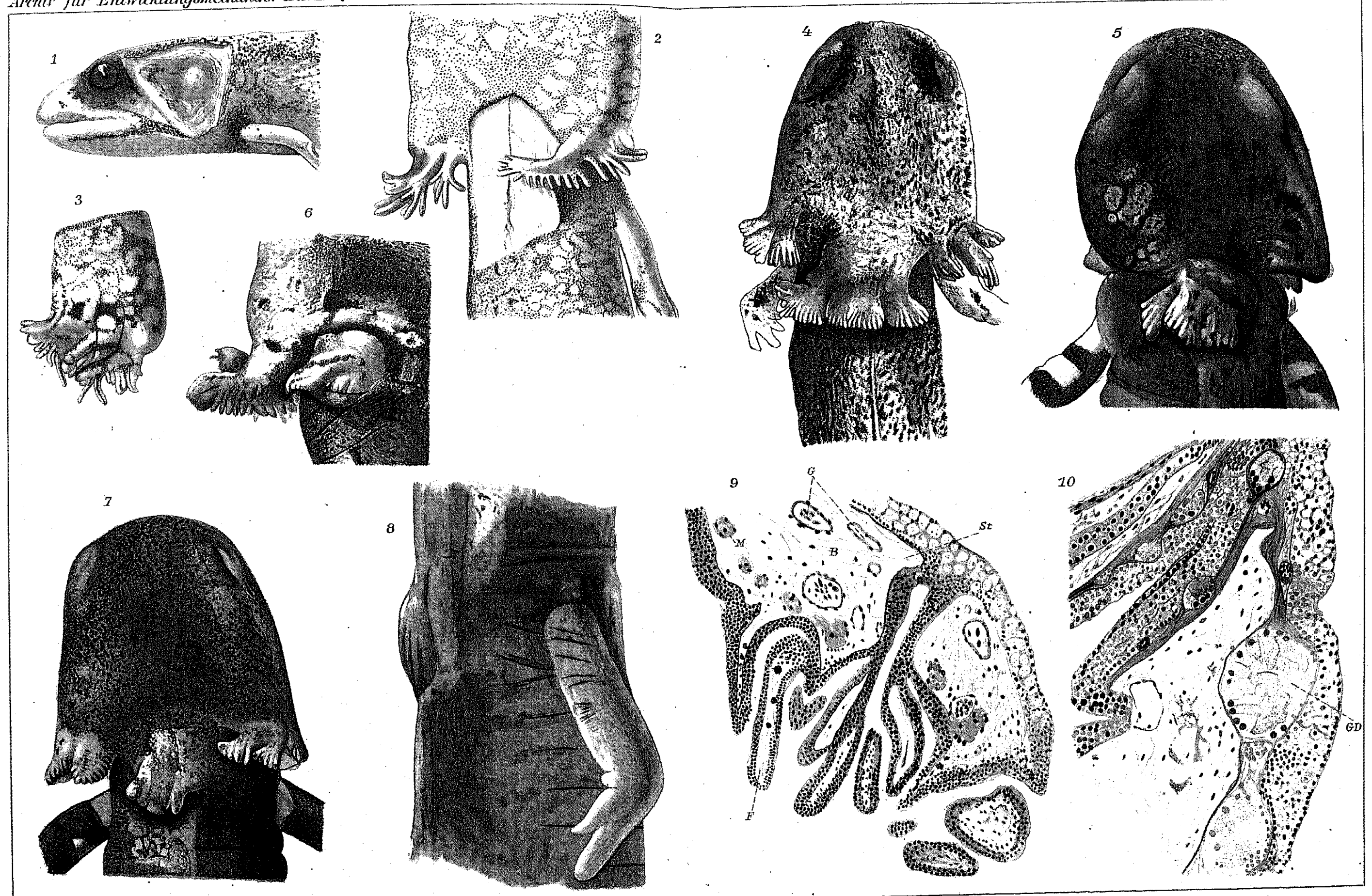




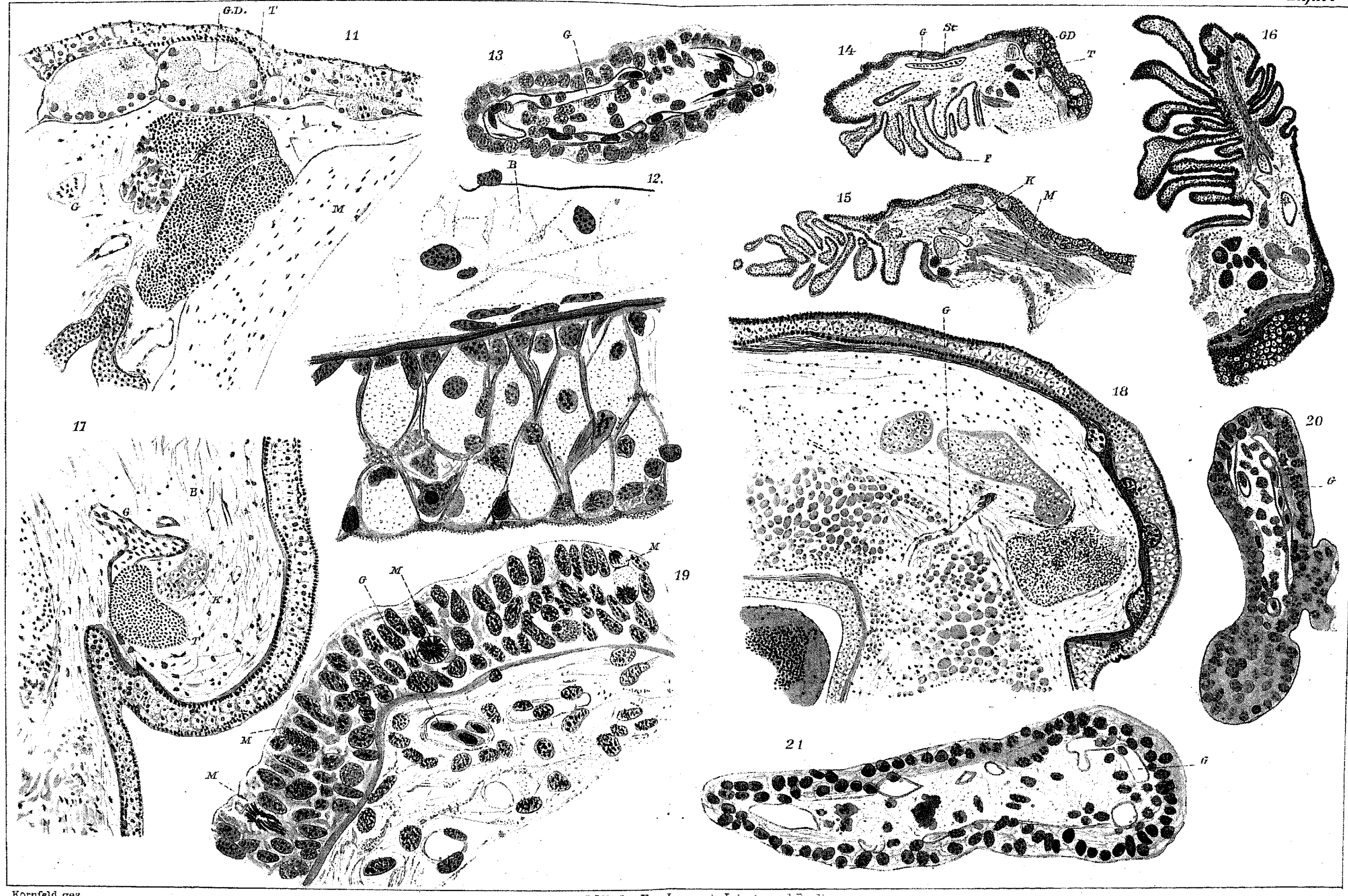


Die Objekte wurden mit Kaliumbichromat-Formol-Eisessig fixiert, mit DeLAFIELDBehem Hämatoxylin-Sïure-Fuchsin-Orange-Alkohol gefürbt.

$$
\text { Fig. 9-13. Normale Ki emen. }
$$

Fig. 9. Übersichtsbild (Stamm, Fiedern, Blutgefüße, Muskeln, Bindegewebe, Bau der Epithelien).

Fig. 10. Ansatzstelle, Giftdriisen.

Fig. 11. Ansatzstelle, Giftdrïsen, Thymus, Muskeln, Blutgefäße.

Fig. 12. Kiemenstamm, dorsales Epithel.

Fig. 13. Fiederquerschnitt mit gefullten Blutgefüßen.

Fig. 14-21. Transplantate.

Fig. 14. Übersichtsbild. Schnitt durch eben abgetragenes Transplantat. (Stamm, Fiedern, Giftdriisen, Blntgefäße, Thymusanschnitte.) Vergr. etwa $18 \times$.

Fig. 15. Ebenso. (Muskeln, Kiemenbogenknorpel.) Vergr. etwa 16×.

Fig. 16. Ebenso.

Fig. 17. Sehnitt durch den Höcker eines im Dauerstadium verharrenden Transplantates. (Bindegewebe, Knorpel, Thymus, BlutgefäBe.)

Fig. 18. Ebenso. Eindringen eines Blutgefäßes ans dem Wirtstier ins Transplantat!

Fig. 19. Epithel eines transplantierten Kiemenstammes im Datterstadium, 4 Mitosen! Gefilltes Blutgefia.

Fig. 20. Querschnitt dureh einen proximalen Teil einer Fieder; geftillte Blutgefüße!

Fig. 21. Quersehnitt durch einen distalen Teil einer Fieder (verödende Blutgefüiße?). 


\section{n. is. \\ - \\ ARCHIV \\ FÜR \\ ENTWICKLUNGSMECHANIK DER ORGANISMEN \\ HERAUSGEGEBEN \\ VON \\ WILHELM ROUX}

o. Ö. PROFESSOR DER ANATOMIE IN HALLE A/S. DR. MED. U. DR. PHIL. HON, C.

\section{VIERZIGSTER BAND}

MIT 90 TEXTFIGUREN UND 22 TAFELN

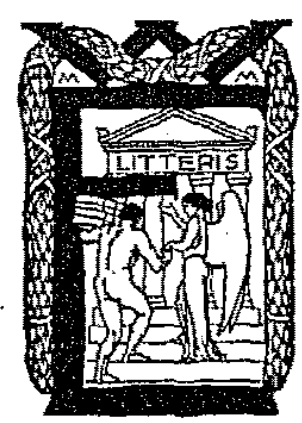

LEIPZIG UND BERLIN

VERLAG VON WILHELM FNGELMANN 